\title{
Design \& Optimization of Large Cylindrical Radomes with Subcell and Non-Orthogonal FDTD Meshes Combined with Genetic Algorithms
}

\author{
Enrique A. Navarro ${ }^{1, *(1)}$, Jorge A. Portí ${ }^{2}{ }^{\circledR}$, Alfonso Salinas ${ }^{3}$, Enrique Navarro-Modesto ${ }^{4}$, \\ Sergio Toledo-Redondo ${ }^{5}$ and Jesús Fornieles ${ }^{3}$ (i) \\ 1 IRTIC Institute/ETSE, Universitat de València, Avd. Universitat, s/n, 46100 Burjassot, Spain \\ 2 Department of Applied Physics, University of Granada, Avd. Fuente Nueva, 16, 08019 Granada, Spain; \\ jporti@ugr.es \\ 3 Department of Electromagnetism and Matter Physics, University of Granada, Avd. Fuente Nueva, 16, \\ 08019 Granada, Spain; asalinas@ugr.es (A.S.); jforniel@ugr.es (J.F.) \\ 4 EPS de Gandia, Universitat Politècnica de València, Calle Paranimf, 1, 46730 Gandia, Spain; \\ ennamo@epsg.upv.es \\ 5 Department of Electromagnetism and Electronics, Campus de Espinardo, University of Murcia, \\ 30100 Murcia, Spain; sergio.toledo@um.es \\ * Correspondence: enrique.navarro@uv.es
}

check for

updates

Citation: Navarro, E.A.; Portí, J.A.; Salinas, A.; Navarro-Modesto, E.; Toledo-Redondo, S.; Fornieles, J. Design \& Optimization of Large Cylindrical Radomes with Subcell and Non-Orthogonal FDTD Meshes Combined with Genetic Algorithms. Electronics 2021, 10, 2263. https:// doi.org/10.3390/electronics10182263

Academic Editor: Yosef Pinhasi

Received: 15 August 2021

Accepted: 12 September 2021

Published: 15 September 2021

Publisher's Note: MDPI stays neutral with regard to jurisdictional claims in published maps and institutional affiliations.

Copyright: (C) 2021 by the authors Licensee MDPI, Basel, Switzerland. This article is an open access article distributed under the terms and conditions of the Creative Commons Attribution (CC BY) license (https:// creativecommons.org/licenses/by/ $4.0 /)$.

\begin{abstract}
The word radome is a contraction of radar and dome. The function of radomes is to protect antennas from atmospheric agents. Radomes are closed structures that protect the antennas from environmental factors such as wind, rain, ice, sand, and ultraviolet rays, among others. The radomes are passive structures that introduce return losses, and whose proper design would relax the requirement of complex front-end elements such as amplifiers. The radome consists mostly in a thin dielectric curved shape cover and sometimes needs to be tuned using metal inserts to cancel the capacitive performance of the dielectric. Radomes are in the near field region of the antennas and a full wave analysis of the antenna with the radome is the best approach to analyze its performance. A major numerical problem is the full wave modeling of a large radome-antenna-array system, as optimization of the radome parameters minimize return losses. In the present work, the finite difference time domain (FDTD) combined with a genetic algorithm is used to find the optimal radome for a large radome-antenna-array system. FDTD uses general curvilinear coordinates and sub-cell features as a thin dielectric slab approach and a thin wire approach. Both approximations are generally required if a problem of practical electrical size is to be solved using a manageable number of cells and time steps in FDTD inside a repetitive optimization loop. These approaches are used in the full wave analysis of a large array of crossed dipoles covered with a thin and cylindrical dielectric radome. The radome dielectric has a thickness of $\sim \lambda / 10$ at its central operating frequency. To reduce return loss a thin helical wire is introduced in the radome, whose diameter is $\sim 0.0017 \lambda$ and the spacing between each turn is $\sim 0.3 \lambda$. The genetic algorithm was implemented to find the best parameters to minimize return losses. The inclusion of a helical wire reduces return losses by $\sim 10 \mathrm{~dB}$, however some minor changes of radiation pattern could distort the performance of the whole radome-array-antenna system. A further analysis shows that desired specifications of the system are preserved.
\end{abstract}

Keywords: radomes; FDTD; sub-cell features; curvilinear coordinates; Genetic Algorithms

\section{Introduction}

This work presents the design procedure of a large radome, to cover an array with a large number of antennas, up to 48 , whose height is of the order of 24 wavelengths and with small elements for adaptation of the order of 0.002 wavelengths. We present the technique we consider most suitable, finite difference time domain (FDTD), with sub-cell 
elements and curvilinear coordinates. This technique is combined with an optimization process based on a genetic algorithm, and the combination of procedures proves to be appropriate for several specific cases.

A major issue in the antenna development is the final operation when the antenna is embedded in the device or the communication equipment. The antenna is not in open space, usually is covered with a case, wrapped in a large structure, or protected by a plastic radome which may be designed as a polarizer rotator or frequency filter [1,2]. The word Radome is a contraction of radar and dome. The function of radomes is to protect antennas from atmospheric agents. Radomes are closed structures that protect the antennas from environmental factors such as wind, rain, ice, sand, and ultraviolet rays, water, [3] among others. Another function of radomes is to hide the antennas or camouflage them to prevent them from being seen. Radomes have different geometries to adapt to the antenna they cover [4] and are usually formed by laminar structures of synthetic materials with good electrical and mechanical properties $[5,6]$. The geometrical shape of the radome must not only be adapted to protect the antenna, [7], it must also be properly combined with the materials and geometry to allow a good adaptation of the signal emitted by the antenna [8]. In short, protecting the antenna has a cost in signal loss, and in the design process an optimal result must be pursued so that there is a good combination of protection and signal transmission. The radomes are passive structures whose proper design would relax the requirement of front-end elements such as amplifiers; an improvement on transmission reduces the gain requirement on the transmitter RF power amplifier, which is a complex active device. Similarly, on the receiver side, it can reduce the stringent noise figure requirement of the low-noise amplifier.

The radome consists mostly in a thin dielectric curved shape cover that usually needs to be tuned using metal inserts or other elements to cancel the capacitive performance of the dielectric [9]. The radome introduces return losses and to obtain good matching metal inserts with inductive behavior can be introduced to minimize reflection to the antenna, with a minimum alteration of radiated fields to the open space [1]. The matching would be carried out with a thin metal wire that would introduce an inductive reactance that would compensate the capacitive behavior of the dielectric slab [10]. The cancellation of an inductive region with a capacitive one is the idea behind other works in which the wave transmission through dielectric layers is improved [11-13].

Radomes are in the near field region of the antenna and approximated circuit techniques [14] or far field formulations [15] do not provide sufficient accuracy for a proper design to minimize return losses. A full wave analysis of the antenna covered with the radome gives the best approach in order to avoid future corrections to the final prototype, with expensive measurements in the anechoic chamber, [16,17]. The FDTD method has been used to solve a large variety of full wave electromagnetic problems over the last 35 years [18]. These include scattering and interaction problems, [19,20], microwave passive devices [21,22], complex shaped tapers [23] and antennas [24,25], among others [18]. The size and complexity of the structures under study have increased as computational capabilities have grown. However, FDTD formulations have always tended to reduce the computer requirements, to optimize the use of computer resources, for instance using predictors to reduce the simulation time in lossless high Q structures [26], or implementing predictive neural networks [27]. By nature, the FDTD method is computationally intensive and approximations are generally required if a problem of practical electrical size has to be solved with a manageable number of cells and time steps. Examples of such approximations include radiation boundary conditions or absorbing boundary conditions [28,29], impedance relationships between field components, and sub cell details in the FDTD modeling [30,31]. A comparison between several different sub cell techniques to model thin dielectric shell and metal sheets is found in [32]. The modeling of cylindrical thin wires is also demanding in terms of computer resources, unless some sort of static field approach similar to that found in [32] is used. 
The main issue in FDTD calculations is the correct introduction of boundary conditions. FDTD solves the Maxwell's curl equations and boundary conditions must describe the structure under study, otherwise the numerical results are not correct. Because typical FDTD technique uses a rectangular cell, curved boundaries are modeled with a staircase shape [25] approaching the true curved boundary. When curved boundaries have a small radius a large mesh is necessary. The use of curvilinear coordinates circumvents the staircase issue [29], however, the number of field components is duplicated with the introduction of covariant and contravariant components [33,34]. Furthermore, new matrices are needed to obtain contravariant components from covariant ones. The metric tensor associated to each curvilinear cell is the matrix that transforms covariant into contravariant components. The main advantage of curvilinear cells is the easy way to apply the boundary conditions, which are introduced in a simple natural manner [29].

The choice between a simple rectangular cell with simple updating FDTD equations and a sophisticated FDTD with a large number of matrices and memory allocation would depend on the shape and geometry of the boundary conditions of the structure under study. There is a trade-off between memory consumption versus expected accuracy, and this is strongly related to geometrical complexity of the modelled antenna. For example, planar antennas are always efficiently managed using rectangular cells [24], and also quasi planar antennas [25].

In this work, the FDTD in curvilinear coordinates is revisited, [16,34], in order to show that it is a powerful tool in the full wave analysis of the radome-antenna structure. The curvilinear FDTD permits the establishment of boundary conditions in arbitrarily shaped surfaces, in an easy and natural way. Moreover, we introduce non-orthogonal sub-cell features to include thin dielectric slabs and thin wires. These sub cell features, which are conveniently inserted in the FDTD algorithm, increase the efficiency of the FDTD implementations because they considerable reduce the number of cells required to give a proper resolution to the radome thickness and metal inserts used for its tuning.

With the proposed technique we face the three-dimensional electromagnetic modeling of a large radome-antenna-array system. The technique is employed in the analysis of an array of 20 to 48 crossed dipoles [35] separated $\lambda / 2$, covered with a thin cylindrical dielectric radome. The thickness of the radome is negligible in terms of wavelength, the thickness below $\lambda / 10$, and dimensions of the overall structure, whose height is around $24 \lambda$, $11.28 \mathrm{~m}$ at the central frequency of the working band. Although the proposed technique is powerful in the analysis of radome-antenna structures, there is still a major problem: the challenge to find the optimal metal insert that maximizes the overall transmission for a given frequency band. In our case, it is proposed to introduce a helical metal wire of diameter $\sim 0.002 \lambda$ in the dielectric cover to cancel the capacitive behavior of the dielectric.

The FDTD code running on a Graphical Processing Unit (GPU) is fast enough to be combined with an optimization algorithm [36], and we use a genetic algorithm (GA) as optimization algorithm. GAs are optimization algorithms inspired by natural selection processes observed in nature [37]. GAs are a powerful tool when searching for an optimal or semi-optimal solution in complex optimization problems [38-40], typically used in phased array design [41], or circuits [42]. A large number of comercial software packages exist also as open sources, however is difficult to find reliable FDTD or finite element method (FEM) software with this numerical power: Curvilinear mesh, sub-cell features, and optimization procedures for design purpose. This is the case, for example, of Ansys using Finite Element Method (FEM), [43,44]. There is no optimization procedure, and it works frequency by frequency, in FDTD we obtain results in the whole band using the Fast Fourier Transform. In Ansys the design optimization is by hand, we have to modify the boundaries gently until we obtain the desired response, and finding a proper design is not easy [43]. The programs used are our own, programmed by us since almost the beginning of the FDTD technique in the last 30 years, in Fortran, C, and Matlab, these have been updated and improved over time [18]. 
This manuscript is organized as follows. Section 2 revisits the curvilinear FDTD technique. The objective of this section is to present the curvilinear FDTD technique in a clear and pedagogical way, providing the information of managed matrices and memory allocation. Section 3 presents the sub-cell dielectric features that are introduced in the original curvilinear FDTD technique. The sub-cell correction of static field in the proximity of a thin wire is presented in Section 4 .

Section 5 presents the GA optimization algorithm to obtain the best parameters for minimizing return losses and, finally, results, analysis and discussion are presented in Section 6 for a Radome-Array of Crossed Dipoles system (RACD) working at UHF frequencies.

\section{The Curvilinear FDTD}

The curvilinear finite difference time domain technique, [12,13], is deployed in a general curvilinear mesh with non-orthogonal cells. The mesh is defined with coordinates $\left(u^{1}\right.$, $\left.u^{2}, u^{3}\right)$, see Figure 1, specially developed for the structures under study, i.e., the boundary conditions are imposed along $u^{1}, u^{2}$, or $u^{3}$ lines or $u^{i}, u^{j}$, planes $(i, j=1,2,3)$. The cylindrical or spherical coordinates are special cases of the general curvilinear coordinates [12]. The curvilinear mesh can be related to an orthogonal Cartesian mesh through a mapping $Q$ that establishes a relationship between the nodes $\left(u^{1}, u^{2}, u^{3}\right)$ and a Cartesian coordinate system $(x, y, z)$, that is $\left(u^{1}, u^{2}, u^{3}\right)=\left(u^{1}(x, y, x), u^{2}(x, y, z), u^{3}(x, y, z)\right)$. The discretization of the curvilinear mesh is similar to the one of orthogonal mesh, by setting the vertices of each cell along the $\left(u^{1}, u^{2}, u^{3}\right)$ directions. The difference with orthogonal FDTD is the cell: in a curvilinear mesh the cell is a hexahedron, a volume enclosed in six faces with twelve edges and eight vertices.
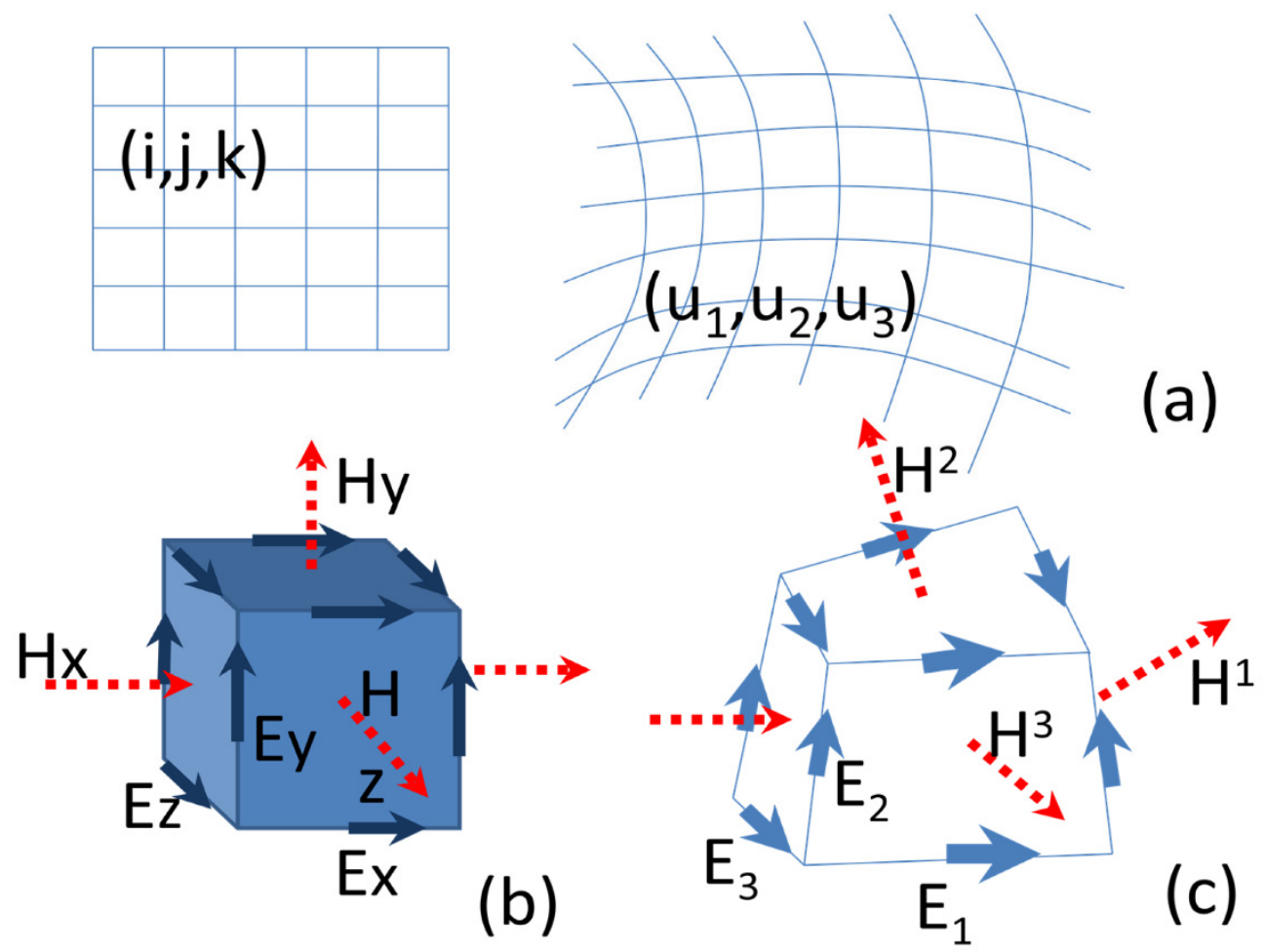

Figure 1. (a) Curvilinear mesh. (b) FDTD orthogonal cell. (c) Covariant and contravariant fields in curvilinear cell.

When the Maxwell's curl equations are enforced at each curvilinear cell (Figure 1c) two sets of vectors are necessary: the vectors that are along the edges of the cell, and the vectors that are perpendicular to the faces. 
The Faraday's law circulates the electric field components along the edges of a face to calculate the time derivative of the magnetic field. The calculated magnetic field component is perpendicular to the surface enclosed by the circulation path. The electric field components along the edges are covariant components, and the magnetic field components are contravariant components. Contravariant components are perpendicular to the faces of the hexahedron, therefore are not aligned along the cell edges in general curvilinear meshes.

The Ampère's law circulates the magnetic field components along the edges of each face, these are the covariant magnetic field components, used to calculate the time derivative of the electric field orthogonal to circulation plane, in doing this, the contravariant electric field components are obtained.

The implementation of Curvilinear FDTD differs from rectangular FDTD in the use of covariant and contravariant components, inherent to a hexahedron cell. In rectangular FDTD, covariant and contravariant components are coincident because at every cell one of the edges is perpendicular to the surface defined by the other two edges. This is not the case when general hexahedron cells are used [29]. Twice the field components are necessary in curvilinear FDTD when compared with rectangular FDTD. However, with a curvilinear mesh the boundary conditions are set very accurately using a coarse mesh; and a coarse mesh is a smaller mesh that consumes lower computer resources. Thus, if the expected curvilinear mesh is smaller than half the equivalent rectangular mesh, the use of non-orthogonal cells is justified.

In curvilinear FDTD the covariant fields are needed to calculate the contravariant fields, and extra computer resources in terms of calculation and memory allocation are necessary in its implementation:

Extra memory in curvilinear FDTD:

- $\quad$ Covariant and contravariant fields at each cell node

- Metric tensor $g_{i j}$, is a $3 \times 3$ symmetrical matrix defined each cell to carry out contravariant to covariant transformation

- Calculation effort in terms of CPU/GPU in curvilinear FDTD

- Contravariant to covariant transformation using the metric tensor $g_{i j}$

Contravariant fields must be down-converted to covariant ones and this requires a matrix operation [33] using the metric tensor of the mesh, $g_{i j}$. These matrices are position dependent in a general curvilinear mesh and have to be calculated at each cell. The memory requirements are increased in comparison with a rectangular scheme. The choice between both techniques should analyze a trade-off accuracy + computer + complexity. There is an extra element to make the choice, the complexity in doing a curvilinear mesh to fit the device under study. These are the key factors to decide whether to use a very fine and large rectangular mesh, or a coarse and small, but complex, conformal mesh.

The mesh points are given in the mesh file. Indexing them $(i, j, k)$ as a numbering of adjacent cells along the lines of the mesh, the $(i, j, k)$ indexing follows the numbering of a Cartesian mesh, and the mesh points are defined as a mapping between the Cartesian index and the mesh points:

$$
\left[i=1 \ldots n_{1}, j=1 \ldots n_{2}, k=1 \ldots n_{3}\right] \mapsto\left[\vec{r}\left(u_{1}(i, j, k), u_{2}(i, j, k), u_{3}(i, j, k)\right)\right]
$$

The covariant basis vectors are obtained at each node of the FDTD mesh along the cell edges, or mesh lines (see Figure 1),

$$
\vec{A}_{i}=\frac{\partial}{\partial u^{i}} \vec{r}\left(u^{1}, u^{2}, u^{3}\right) \approx \frac{\Delta \vec{r}\left(u^{1}, u^{2}, u^{3}\right)}{\Delta u^{i}}
$$

And the contravariant vectors are normal to the FDTD cell faces.

$$
\vec{A}^{1}=\frac{1}{\sqrt{g}} \cdot\left(\vec{A}_{2} \times \vec{A}_{3}\right),
$$




$$
\begin{aligned}
& \vec{A}^{2}=\frac{1}{\sqrt{g}} \cdot\left(\vec{A}_{3} \times \vec{A}_{1}\right), \\
& \vec{A}^{3}=\frac{1}{\sqrt{g}} \cdot\left(\vec{A}_{1} \times \vec{A}_{2}\right),
\end{aligned}
$$

The metric tensor is:

$$
g_{i j}=\vec{A}_{i} \times \vec{A}_{j}
$$

$g$ is cell volume:

$$
\sqrt{g}=\vec{A}_{1} \cdot\left(\vec{A}_{2} \times \vec{A}_{3}\right)
$$

The covariant and contravariant formulation of Maxwells's curls equations arises natural from original Ampère and Faraday-Henry Laws in integral form [16] in general curvilinear coordinates. The contravariant components of the electric field are obtained by projecting the curl on the $\vec{A}^{i}$, contravariant vectors:

$$
(\nabla \times \vec{E}) \cdot \vec{A}^{1}=\frac{1}{\sqrt{g}}\left[\frac{\partial}{\partial u^{2}}\left(\vec{E} \cdot \vec{A}_{3}\right)-\frac{\partial}{\partial u^{3}}\left(\vec{E} \cdot \vec{A}_{2}\right)\right]
$$

The two remaining components have similar expressions, and are obtained from Equation (6) by permutation of indices.

$$
(\nabla \times \vec{E}) \cdot \vec{A}^{i}=\frac{1}{\sqrt{g}}\left[\frac{\partial}{\partial u^{j}}\left(\vec{E} \cdot \vec{A}_{k}\right)-\frac{\partial}{\partial u^{k}}\left(\vec{E} \cdot \vec{A}_{j}\right)\right]
$$

The contravariant $\left(E^{i}, H^{i}\right)$ and covariant $\left(E_{j}, H_{j}\right)$ field components are obtained by projecting the corresponding vectors on the corresponding basis vectors:

$$
\begin{aligned}
& E^{i}=\vec{E} \cdot \vec{A}^{i} \\
& E_{j}=\vec{E} \cdot \vec{A}_{j}
\end{aligned}
$$

Following Equation (7), is obtained the following expression involving the contravariant electric field components $E^{1}$, and the $H_{2}, H_{3}$ covariant components:

$$
\frac{\partial E^{1}}{\partial t}=\frac{1}{\varepsilon \sqrt{g}}\left[\frac{\partial}{\partial u^{2}} H_{3}-\frac{\partial}{\partial u^{3}} H_{2}\right]
$$

Similar expressions are derived for the other field components $E^{2}, E^{3}$ by index permutation.

$$
\frac{\partial E^{i}}{\partial t}=\frac{1}{\varepsilon \sqrt{g}}\left[\frac{\partial}{\partial u^{j}} H_{k}-\frac{\partial}{\partial u^{k}} H_{j}\right]
$$

From Faraday's law, the following expression is derived that relates $H^{1}$ with $E_{2}, E_{3}$.

$$
\frac{\partial H^{1}}{\partial t}=-\frac{1}{\mu \sqrt{g}}\left[\frac{\partial}{\partial u^{2}} E_{3}-\frac{\partial}{\partial u^{3}} E_{2}\right]
$$

Furthermore, similar expressions are derived for the other field components $H^{2}, H^{3}$, by index permutation.

$$
\frac{\partial H^{i}}{\partial t}=-\frac{1}{\mu \sqrt{g}}\left[\frac{\partial}{\partial u^{j}} E_{k}-\frac{\partial}{\partial u^{k}} E_{j}\right]
$$

Equations (10) and (12) are discretized using the centered difference scheme of FDTD, with the standard notation:

$$
F\left(i \Delta u_{1}, j \Delta u_{2}, k \Delta u_{3}, n \Delta t\right)=F^{n}(i, j, k)
$$


The basis vectors are obtained, which lately are used to calculate $g_{i j}$ :

$$
\begin{aligned}
& \vec{A}_{1}=\vec{r}(i, j, k)-\vec{r}(i-1, j, k) \\
& \vec{A}_{2}=\vec{r}(i, j, k)-\vec{r}(i, j-1, k) \\
& \vec{A}_{3}=\vec{r}(i, j, k)-\vec{r}(i, j, k-1)
\end{aligned}
$$

Discretization of Equations (10) and (12) is as follows for $E^{1}, H^{1}$, and similarly for $E^{2-3}, H^{2-3}$ by index rotation:

$$
\begin{aligned}
& E^{1}(i, j, k)^{n+1}=E^{1}(i, j, k)^{n}+\frac{\Delta t}{\varepsilon \sqrt{g}}\left[\begin{array}{l}
H_{3}(i, j, k)-H_{3}(i, j-1, k) \\
-H_{2}(i, j, k)+H_{2}(i, j, k-1)
\end{array}\right]^{(n+1 / 2)} \\
& H^{1}(i, j, k)^{n+1 / 2}=H^{1}(i, j, k)^{n-1 / 2}-\frac{\Delta t}{\mu \sqrt{g}}\left[\begin{array}{l}
E_{3}(i, j+1, k)-E_{3}(i, j, k) \\
-E_{2}(i, j, k+1)+E_{2}(i, j, k)
\end{array}\right]^{n}
\end{aligned}
$$

To stagger the Ampere and Faraday laws in the numerical FDTD scheme, the conversion from contravariant to covariant components is required, and this conversion is carried out by means of the metric tensor $g_{i j}$ [33], where Einstein notational convention of summation over repeated indexes is followed:

$$
\begin{gathered}
E_{j}=g_{j i} \cdot E^{i} \\
H_{j}=g_{j i} \cdot H^{i}
\end{gathered}
$$

The FDTD-GCC implementation is summarized in the following steps:

- Initial values: Variables are initialized and constant parameters are defined.

- Contravariant field components $E^{i}$ are obtained using Ampere's Law, Equation (15) and similarly for $E^{2-3}$ by index rotation.

- Calculation of the covariant $E_{i}$, components from the contravariant $E^{i}$ with (17a).

- Boundary conditions, and stimulus on covariant field components are set.

- Sampling, storing and processing of $E_{i}$ fields.

- Contravariant fields $H^{i}$ are obtained from covariant fields $E_{i}$, by using (16) and similarly for $\mathrm{H}^{2-3}$ by index rotation.

- Calculation of the covariant $H_{i}$, components from the contravariant $H^{i}$ with (17b).

- Boundary conditions on covariant components $H_{i}$ are set.

- $\quad$ Sampling, storing and processing of $H_{i}$ fields.

An important parameter to keep the stability of the time marching FDTD is the choice of time step $\Delta t$. Some derivations for the Courant condition in curvilinear coordinates have been addressed in our previous work $[17,29]$ :

$$
\Delta t=\frac{\min \left(\sqrt{g_{m m}(i, j, k)}\right)}{2 c}, \quad m=1,2,3 ; \quad(i, j, k)=(1: n 1,1: n 2,1: n 3)
$$

\section{Sub-Cell Modeling of a Thin Dielectric Slab with Losses}

We assume a thin dielectric slab located along the surface $u_{2}-u_{3},\left(u_{1}=\right.$ constant). On this, the equations for the field components crossing the slab are modified. The model introduces a new and additional field node located at the position of the thin slab, adapted to the special cell [16]. The slab has thickness $d$ in the $u_{1}$ direction (see Figure 2). 


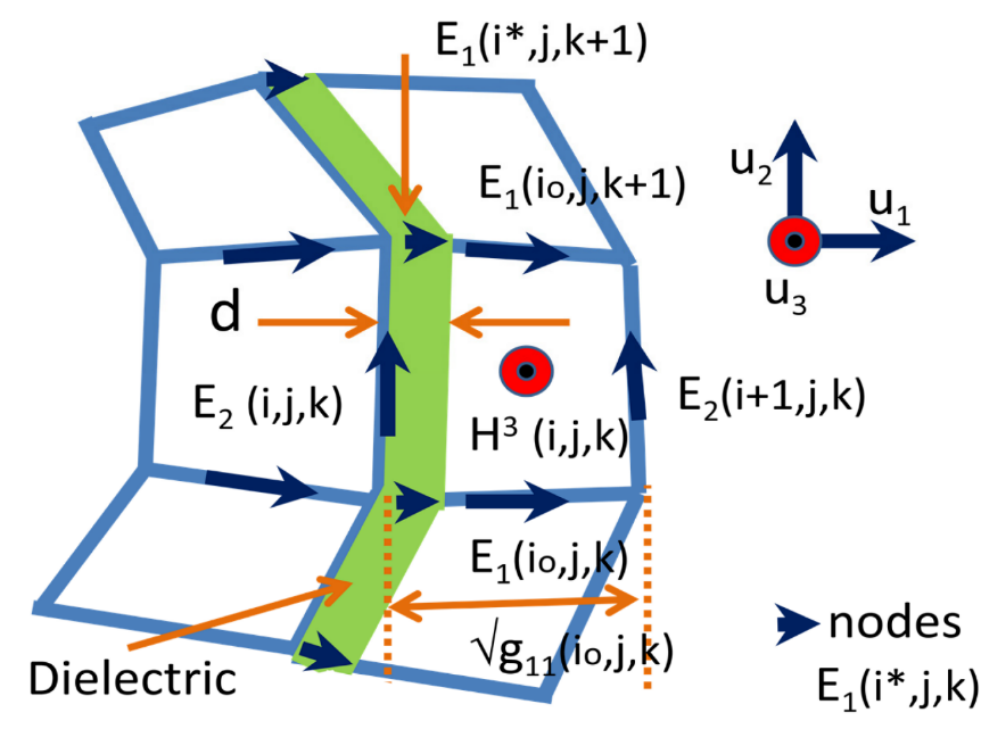

Figure 2. Special subgrid model of a thin dielectric slab. Index $\left(^{*}\right)$ for special nodes.

The thin material slab has conductivity $\sigma$, dielectric constant $\varepsilon$, and permeability $\mu=\mu_{0}$. The above FDTD procedure is used for all the cells with exception of the special cells where the thin slab is located. In these special cells, the electric field normal to the slab, $E^{1}$, is split in sub-components $E^{1}\left(i^{*}, j, k\right)$ and $E^{1}\left(i_{0}, j, k\right)$, to take in account the discontinuity in the dielectric. The $\mathrm{i}_{0}$, accounts for the line $u_{1}=$ constant, where the thin slab is located, and $i^{*}$ is the index of the new field node located at the position of the thin slab. The other field components do not need to be split because they are tangential to the slab and are continuous functions. The update equations for $E^{1}\left(i_{0}, j, k\right)$ involve $H_{3}$ and $H_{2}$ that are outside the dielectric. The $E^{1}\left(i^{*}, j, k\right)$, located in the special nodes of the dielectric are updated using the same magnetic field components as $E^{1}\left(i_{0}, j, k\right)$, because the $H_{3}$ and $H_{2}$ components are continuous across the dielectric. However, the closed path of Ampere's law used in the updating of $E^{1}\left(i^{*}, j, k\right)$ is inside the dielectric. Therefore, when calculating $E^{1}\left(i^{*}, j, k\right)$, dielectric constant changes and losses are considered,

$$
E^{1}\left(i^{*}, j, k\right)^{n+1}=\frac{1-\Delta t \sigma / 2 \varepsilon}{1+\Delta t \sigma / 2 \varepsilon} E^{1}\left(i^{*}, j, k\right)^{n}+\frac{\Delta t / \varepsilon}{1+\Delta t \sigma / 2 \varepsilon} \frac{1}{\sqrt{g}}\left[\begin{array}{l}
H_{3}(i, j, k)-H_{3}(i, j-1, k) \\
-H_{2}(i, j, k)+H_{2}(i, j, k-1)
\end{array}\right]^{(n+1 / 2)}
$$

The updated equations for the electric fields tangential to the slab are obtained by considering that only a portion $d$ of the enclosed path is inside the material, the factor $f$ is defined as:

$$
f=\frac{d}{\Delta u_{1}}\left(\frac{\partial r}{\partial u_{1}}\right)^{-1}=\frac{d}{\sqrt{g_{11}\left(i^{*}, j, k\right)}}
$$

$E^{2}$, and $E^{3}$, are obtained using the modified conductivity and permittivity $\sigma_{m}, \varepsilon_{m}$ :

$$
\begin{gathered}
\sigma_{m}=f \sigma \\
\varepsilon_{m}=(1-f) \varepsilon_{0}+f \mathcal{\varepsilon}
\end{gathered}
$$

Equations for $E^{2}$, and $E^{3}$ are:

$$
\begin{aligned}
& E^{2}(i, j, k)^{n+1}=\frac{1-\Delta t \sigma_{m} / 2 \varepsilon_{m}}{1+\Delta t \sigma_{m} / 2 \varepsilon_{m}} E^{2}(i, j, k)^{n}-\frac{\Delta t / \varepsilon_{m}}{1+\Delta t \sigma_{m} / 2 \varepsilon_{m}} \frac{1}{\sqrt{g}}\left[\begin{array}{l}
H_{3}(i, j, k)-H_{3}(i-1, j, k) \\
-H_{1}(i, j, k)+H_{1}(i, j, k-1)
\end{array}\right]^{(n+1 / 2)} \\
& E^{3}(i, j, k)^{n+1}=\frac{1-\Delta t \sigma_{m} / 2 \varepsilon_{m}}{1+\Delta t \sigma_{m} / 2 \varepsilon_{m}} E^{3}(i, j, k)^{n}+\frac{\Delta t / \varepsilon_{m}}{1+\Delta t \sigma_{m} / 2 \varepsilon_{m}} \frac{1}{\sqrt{g}}\left[\begin{array}{l}
H_{2}(i, j, k)-H_{2}(i-1, j, k) \\
-H_{1}(i, j, k)+H_{1}(i, j-1, k)
\end{array}\right]^{(n+1 / 2)}
\end{aligned}
$$

The new field component $E^{1}\left(i^{*}, j, k\right)$ is located in the cell fraction that contains the dielectric slab, and is introduced in the equations where this lies in the closed surrounding 
path of Ampere's law. Thus, the equation for $H^{1}$ is not modified (normal to the slab). However, $H^{2}$, and $H^{3}$, (tangential to the slab) include the new field component $E^{1}\left(i^{*}, j, k\right)$, because it lies in the closed surrounding path that contains the slab.

$$
\begin{aligned}
& H^{2}(i, j, k)^{n+1 / 2}=H^{2}(i, j, k)^{n-1 / 2}-\frac{\Delta t}{\mu \sqrt{g}}\left[\begin{array}{l}
(1-f) \cdot\left(E_{1}\left(i_{0}, j, k+1\right)-E_{1}\left(i_{0}, j, k\right)\right) \\
+f \cdot\left(E_{1}\left(i^{*}, j, k+1\right)-E_{1}\left(i^{*}, j, k\right)\right) \\
-E_{3}(i+1, j, k)+E_{3}(i, j, k)
\end{array}\right]^{n} \\
& H^{3}(i, j, k)^{n+1 / 2}=H^{3}(i, j, k)^{n-1 / 2}+\frac{\Delta t}{\mu \sqrt{g}}\left[\begin{array}{l}
(1-f) \cdot\left(E_{1}\left(i_{0}, j+1, k\right)-E_{1}\left(i_{0}, j, k\right)\right) \\
+f \cdot\left(E_{1}\left(i^{*}, j+1, k\right)-E_{1}\left(i^{*}, j, k\right)\right) \\
-E_{2}(i+1, j, k)+E_{2}(i, j, k)
\end{array}\right]^{n}
\end{aligned}
$$

The covariant fields are calculated with the corresponding metric tensor, whereas the special covariant $E^{1}\left(i^{*}, j, k\right)$, is calculated using the metric tensor of $E^{1}\left(i_{0}, j, k\right)$. This approach is consistent with our assumption of a thin material slab having a width much smaller than the cell dimensions.

\section{Thin Wire Sub-Cell Model}

Typical FDTD implementations model wires of finite radius as infinitesimal thin wires. Depending on the structure under study the infinitesimal modeling would give good results or not. Traditionally, the establishment of the hard boundary of zero tangential fields has a slight error of discretization that may lead to small numerical inaccuracies which mainly affect resonant structures, giving rise to small displacements in frequency with respect to experimental results. These small deviations appear in the FDTD analysis of wire structures, or narrow apertures in conductors. Moreover, resistive thin wires have known impedance that depends on the finite radius and conductivity of the wire, which is neglected in case of infinitesimal assumption. One way to take into account the finite radius of the wires, and improve the numerical FDTD results, is the incorporation of the known behavior of the electromagnetic field in the close vicinity of conductors. This is especially important in the treatment of thin wires [45-49], as well as in various matching structures to improve the behavior of the radomes. The electric field is perpendicular near a conducting surface, and the magnetic field rotates around the conductors, both fields having a dominant behavior $1 / r$, where $r$ is the distance to conductor. This is a quasi-static behavior, and its introduction into Maxwell's equations allows the modeling of small conductive structures within the cells.

Let's assume a conductive thin wire of radius $r_{0}$ along the $u_{3}$-axis, being $r_{0}$ much smaller than the cell dimensions. The thin wire is assumed to be in the $u_{3}$-axis, where the $E_{3}\left(i=i_{0}, j=j_{0}, k\right)$ nodes are located (Figure 3$)$; therefore the typical boundary condition in the wire is $E_{3}\left(i_{0}, j_{0}, k\right)=0$. To take into account the finite dimension of the radius $r_{0}$ inside the curvilinear cell, being $r_{0} \ll \sqrt{g_{11}(i, j, k)}$, we assume a quasi-static behavior of the electric and magnetic fields in the proximity of the wire. Near the conductor tangential electric field and normal magnetic field approach 0 , and normal electric field and circulating magnetic field approach static field behavior $1 / r$, where $r$ is the distance to the center of the wire. Therefore electromagnetic fields can be written in terms of known field values and $1 / r$, dependence:

$$
\begin{aligned}
H^{1}\left(i, u_{2}, k\right)^{n+1 / 2} & =H^{2}(i, j, k)^{n+1 / 2} \frac{1}{2 u_{2}} \\
H^{2}\left(u_{1}, j, k\right)^{n+1 / 2} & =H^{2}(i, j, k)^{n+1 / 2} \cdot \frac{1}{2 u_{1}} \\
E_{1}\left(u_{1}, j,(k+1 / 2) \pm 1 / 2\right)^{n} & =E_{1}(i, j,(k+1 / 2) \pm 1 / 2)^{n} \cdot \frac{1}{2 u_{1}}
\end{aligned}
$$




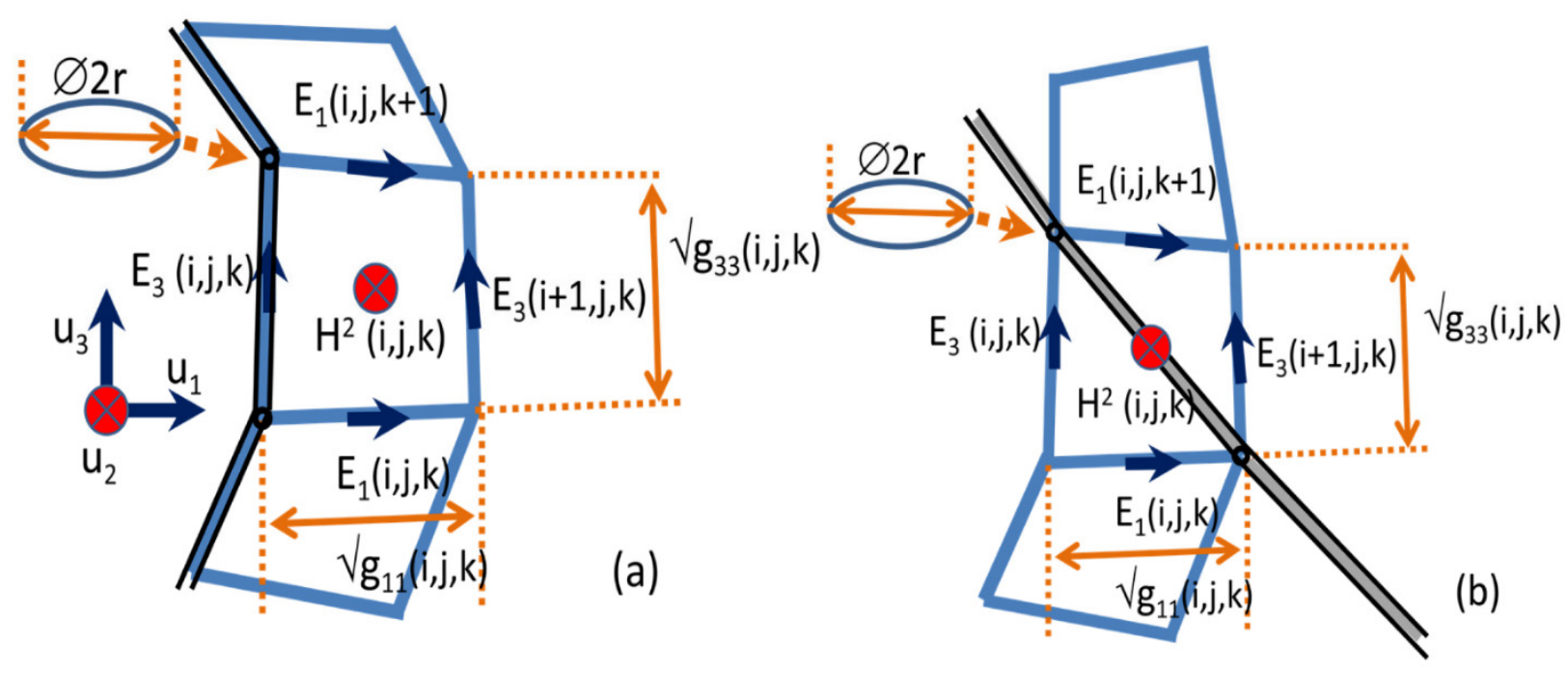

Figure 3. (a) Thin wire along the covariant field. (b) Thin wire oblique to covariant field.

The substitution of the above field dependence in the Maxwell's curl equations produces the following expressions,

$$
\begin{aligned}
& \frac{\partial H^{2}(i, j, k)^{n}}{\partial t} \cdot \frac{\sqrt{g_{11}(i, j, k)}}{2} \cdot \ln \left(\frac{\sqrt{g_{11}(i, j, k)}}{r_{0}}\right) \cdot \sqrt{g_{33}(i, j, k)}= \\
& {\left[E_{1}(i, j, k+1)^{n}-E_{1}(i, j, k)^{n}\right] \cdot \frac{\sqrt{g_{11}(i, j, k)}}{2} \ln \left(\frac{\sqrt{g_{11}(i, j, k)}}{r_{0}}\right)+E_{3}(i+1, j, k)^{n} \cdot \sqrt{g_{33}(i, j, k)}}
\end{aligned}
$$

Equation (24) leads to the update equation for $H^{2}$ :

$$
H^{2}(i, j, k)^{n+1 / 2}=H^{2}(i, j, k)^{n-1 / 2}-\frac{\Delta t}{\mu \sqrt{g}}\left[\begin{array}{l}
E_{1}(i, j, k+1)-E_{1}(i, j, k) \\
+2 E_{3}(i+1, j, k) / \ln \left(\frac{\sqrt{g_{11}(i, j, k)}}{r_{0}}\right)
\end{array}\right]^{n}
$$

A similar expression is derived for the $H^{3}$ contravariant component. The covariant components are calculated from the contravariant components using the same Equation (14), with the metric tensor $g_{i j}$.

\section{Finding the Optimal Radome Design for a Large Antenna array}

Full-wave analysis of large antennas covered by radomes is confronted with the coexistence of large structures with very small elements of reduced dimensions, and these small elements are critical because they are decisive in their operation. The design of a radome for an array of crossed dipoles in the frequency band [470 MHz, $806 \mathrm{MHz}$ ] is proposed. The radome is a cylindrical dielectric thin shell, made on fiber glass, dielectric constant $\varepsilon_{r}=4.25$, whose thickness is a fraction of the wavelength, $\sim \lambda / 10$ in the frequency band, i.e., around 10-20 mm, which is chosen for mechanical and manufacturing reasons. The cylinder has $1600 \mathrm{~mm}$ diameter to comfortably contain the $\lambda / 2$ crossed dipoles. To improve the transmission through the radome an inductive element is introduced, a thin metal wire, whose diameter is also a negligible fraction of the wavelength, which is around $0.002 \lambda$. The use of the technique presented in the previous sections seems perfectly indicated for the analysis of this type of structure, whereas the overall antenna-radome structure has a height of $\sim 24 \lambda$, and a diameter $\sim \lambda$.

The presented technique is powerful and useful in the analysis of the antenna-radome structure, now it remains to address another important problem; to find the optimal radome structure, within the imposed requirements, that will minimize the reflection, or return loss in the given operating band. In our case the objective is to introduce a helical silver wire, conductivity $6.14 \times 10^{7} \Omega^{-1} \cdot \mathrm{m}^{-1}$, having diameter around $0.002 \lambda$, that is $\sim 1 \mathrm{~mm}$ diameter. The helical wire will be wrapped in the external surface of the dielectric shell to cancel the 
capacitive behavior of the dielectric. The inductive behavior of the helical wire is expected to cancel the capacitive behavior of the dielectric shell. The goal is finding the optimum design that maximizes transmission, or conversely, to minimize return losses. Inside the radome, along the cylinder axis, there is an array of 20 to 48 crossed dipoles spaced $\lambda / 2$. The crossed dipoles are transversal to the cylinder axis. Each pair of crossed dipoles is mounted horizontally attached to a common mast. The two dipoles are fed 90 degrees out of phase to generate an omnidirectional pattern. The array structure is expected to radiate broadside with a high gain a horizontal polarized field in the horizontal plane of the crossed dipoles. This antenna itself is expected to provide full coverage of the UHF commercial broadcast bands.

\subsection{Radome Design Strategy Using GA}

Three parameters must be obtained in the optimal design, which will be chosen from among those that give the best radiating behavior of the radome-antenna system. These parameters are:

- $\quad$ Dielectric shell thickness, which will be chosen in the range $15-50 \mathrm{~mm}$, for mechanical and manufacturing reasons.

- Diameter of the silver metallic wire that will constitute the helix that envelops the radome.

- $\quad$ Spacing between turns of the helical wire enveloping the radome.

We use a genetic algorithm (GA) as the optimization algorithm. GAs are optimization algorithms inspired by natural selection processes observed in nature. GAs have been successfully employed in a variety of optimization problems in electromagnetics [37], new algorithms GA are being developed [38], for complex optimization problems [39], in antennas are typically used for array design and optimization [41]. Other techniques could be implemented, but this one works reasonably well and we have tested it in other optimization problems [42].

The GA follows the following scheme:

(1) Random production of an original population whose number of individuals is $\mathrm{N}$.

(2) Produce the next generation by crossovers and mutations among the individuals.

(3) Produce the new population of $\mathrm{N}$ individuals from the generation of (2) by selection.

(4) Produce the next population by repeating steps (2) and (3) until obtaining the individual that satisfies the optimal conditions defined.

In the GA, the individuals of the next population are selected according to the value of the defined fitness function, which is calculated for each individual. In GA, the individuals whose values of the fitness function are higher than the others are kept, and individuals with low fitness are discarded. The value of the fitness function determines the ending condition of the GA search process [41]. If the values of the fitness function do not continue to improve, the algorithm is terminated and the individual with the highest fitness is presented as the optimal solution. Another ending condition of GA is the maximum number of generations, which is also an input. For example, a maximum of 500 generations in the populations can be set, thus another termination condition of the algorithm is when it reaches 500 generations. In that case the algorithm ends and the individual with the highest value of the objective function of the last generation is presented as the optimal solution.

In GA, crossover and mutations are carried out by means of binary $0-1$ operations, therefore the coding of individual parameters is necessary expressing the individual parameters by binary strings of $0 \mathrm{~s}$ and $1 \mathrm{~s}$. In this optimization procedure each Radome-Array of Crossed Dipoles system (RACD) is an individual having three parameters: Thickness, diameter, and spacing. These three design parameters are coded by three 8 - bit string of $0 \mathrm{~s}$ and $1 \mathrm{~s}$. That is, each parameter is a real number and is converted to binary format to set the three chromosomes of the individuals:

Individual $_{i}=(\text { Thickness, } \text { diameter }, \text { spacing })_{i}=(00101111,01010101,11110000)$ 
The GA population has $\mathrm{N}$ individuals as above, each one with three the chromosomes, and each chromosome with eight genes. The objective is to minimize reflection within the operating bandwidth $\left[f_{1}, f_{2}\right]$ of the RACD. We look for a good transmission at the center frequency, which is $f_{c}=\left(f_{1}+f_{2}\right) / 2$. Thus, the fitness function is defined as the average transmission coefficient of the average frequency response in the band with the response at central frequency. In this fitness we give equal importance to the average frequency response over the entire band and the frequency response at the center frequency.

$$
\text { Fitness } \left._{i}=\text { Average }_{\text {Average }}(S 11[f 1 \ldots f 2]), S 11\left[\frac{f 1+f 2}{2}\right]\right)
$$

The objective to minimize will be the average reflection coefficient within the whole band, also giving importance to the center frequency.

The mesh for this optimization procedure is always $42 \times 36 \times 500$ in $u_{1}-u_{2}-u_{3}$ respectively. The FDTD mesh is terminated with Liao Absorbing Boundary Conditions (ABC) [50] because of its simplicity and fast calculation in a curvilinear FDTD mesh. Although PML could be used [51] this are avoided in this modeling to reduce the time spent in the large number of numerical calculations involved in the GA. The reflection coefficient $S_{11}$, return loss, is calculated by using the total-field reflected-field formulation with a synthetic excitation using the near field [21], covering the $\left[f_{1}, f_{2}\right]$ frequency band. The RACD system is designed specifically for UHF broadcast, and the manufacturing specifications are that the radome should minimally alter the original cross-dipole array pattern, especially outside the broadside direction.

\subsection{FDTD-GA Optimization Scheme}

The FDTD program is introduced inside the loop of the GA to calculate the fitness function. The optimization scheme is as follows:

- $\quad$ Creation of a first population of $n=100$ individuals

- $\quad n=100$ random binary 8-bit vectors are created as in Equation (28)

- Binary vectors are converted to decimal values in the given bounds, in this case (Thickness, diameter, spacing) $\in[0.01,0.05] \times[0.10,0.50] \times\left[0.1 \times 10^{-3}\right.$, $1.0 \times 10^{-3}$ ] in meters.

- Fitness is evaluated for each individual:

- Fitness is calculated by running FDTD for each individual (Thickness, diameter, spacing) in this population.

- $\quad$ For $\mathrm{i}=1$ : generation $=\mathrm{G}$, do (searches optimum in $\mathrm{G}=500$ generations)

- Creation of a new population of 100 individual by crossing the individuals of the first population: Two offspring are born from a pair of two individuals, where the pair is randomly selected. There is randomly generated a point for bits (genes) In this way a new population is generated. A point of exchange of genes is randomly chosen to obtain the two offspring of the two parents.

- Mutation: A random number is generated, and if this number is greater than the given probability of mutation $\left(p_{m} \in[0.0,1.0]\right)$, then bit 0 is changed to 1 and bit 1 is changed to zero, in the three randomly generated positions for each chromosome (Thickness, diameter, spacing) in binary.

- Calculation of fitness: $F\left(S_{11}\right)$ is calculated with FDTD for all the individuals of the population.

- Compares individuals in the population to find the one with the best fitness $F\left(S_{11}\right)$. This is compared against previous optimum value, and the best one between them is selected.

- Finds the individual with the worst fitness and this is substituted by the individual with the best fitness. With this process, the individuals with the worst fitness are eliminated from the population and replaced by those with the best fitness. 
- $\quad$ End

- $\quad$ The best individual is obtained by comparison when fitness converges or by the end of generations.

The individuals with the worst fitness are eliminated from the population and replaced by those with the best fitness. The program ends when the best individual is obtained or at the end of the number of generations. In the latter case the optimum value is the best of the final population, taking into account that this population has undergone natural selection and is the one with the highest fitness. Therefore, at the end of the number of generations we could say that the optimum is obtained from the best individual of the best population.

The FDTD-GA optimization scheme was implemented using Matlab ${ }^{\mathrm{TM}} \mathrm{R} 2021 \mathrm{a}$, doing parallelization to take the capabilities of the Graphical Processing Unit (GPU), a NVIDIA GTX-970 GPU card. In doing this the FDTD-GA optimization scheme was able to give a useful design in terms of a few hours, [36].

\section{Numerical Results}

\subsection{Return Loss}

The cylindrical dielectric cover is made on fiberglass, $\varepsilon_{r}=4.25$. Diameter is $1600 \mathrm{~mm}$. The conductivity of the helical wire is $\sigma=6.14 \times 10^{7} \Omega^{-1} \cdot \mathrm{m}^{-1}$, and the other parameter to be found are thickness of dielectric cover, diameter of the wire, and spacing between turns of the helix, which are bounded in the following intervals given in meters: [0.01, 0.05]; $[0.10,0.50]$, and $\left[0.1 \times 10^{-3}, 1.0 \times 10^{-3}\right]$ respectively. The radome completely covers the antenna array, with an extra length of half a wavelength below the first antenna of the array and half a wavelength above the last antenna of the array (see Figure 4).

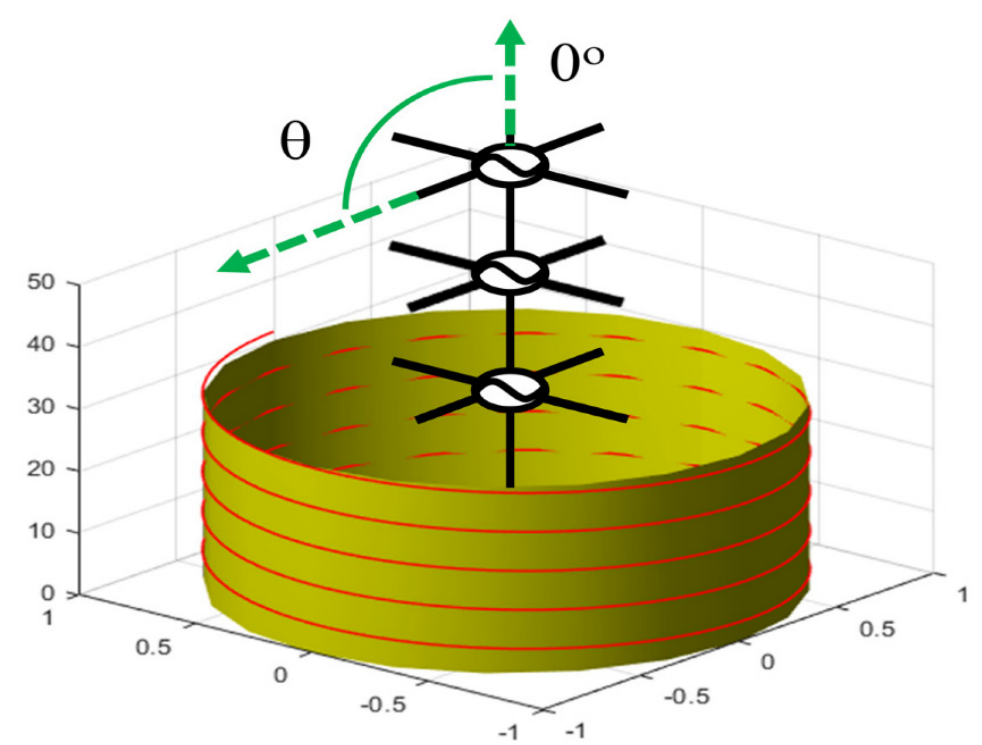

Figure 4. Array of crossed dipoles inside the cylindrical radome.

The FDTD mesh is $42 \times 36 \times 500$ in the directions $\left(u_{1}, u_{2}, u_{3}\right)$, which account for the $(r, \phi, z)$. The overall structure, including the helical wire, has not any symmetry because of the helix. The program runs for $6000 \Delta$ t. Electromagnetic fields inside and outside of the radome are sampled, stored and processed. The excitation is a half sine function with $90^{\circ}$ phase shift between both dipoles. The transmission is calculated as the ratio of the field with radome and without radome, and the return losses accordingly, by subtraction of incident and total field inside the radome. Some preliminary calculations are carried out, and Figures 5 and 6 show the magnitude of the E-field with the radome at a given time instant. Return losses are shown in Figures 7 and 8 for the frequency band under study [470 MHz, $806 \mathrm{MHz}$ ] for different values of the spacing. The return losses for the radome without helical wire are in the interval $[-10.6,-6.5] \mathrm{dB}$, the wrapping helix with spacing 
$\mathrm{s}=0.22 \mathrm{~m}$ improves return losses by more than $10 \mathrm{~dB}$ around $600 \mathrm{MHz}$, and 3-15 $\mathrm{dB}$ in the operating band. This shows that the procedure is adequate, and that it is now feasible to search for an optimal solution.
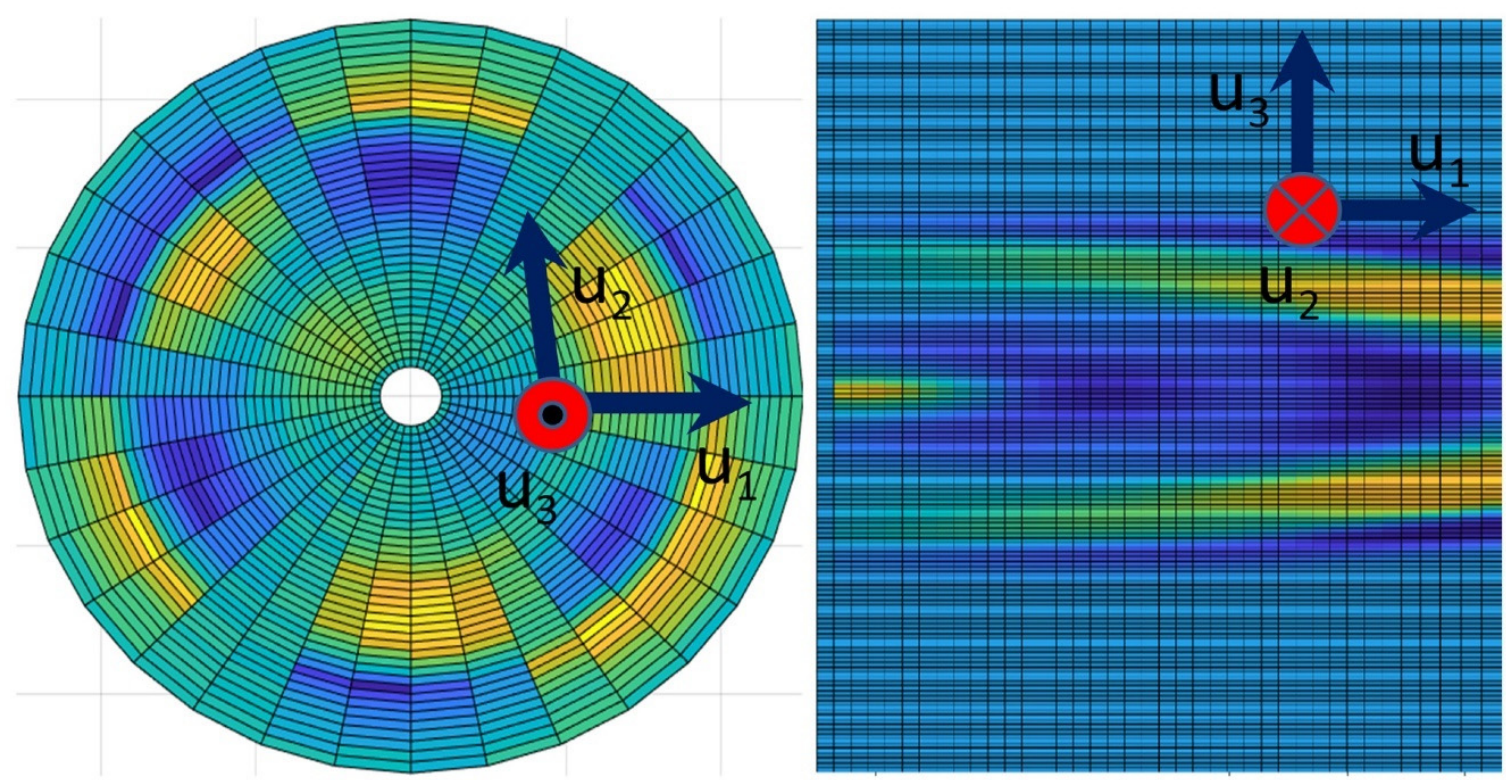

Figure 5. Contour of radiated power in mesh sections $u_{3}=$ constant and $u_{2}=$ constant, at a given time instant.

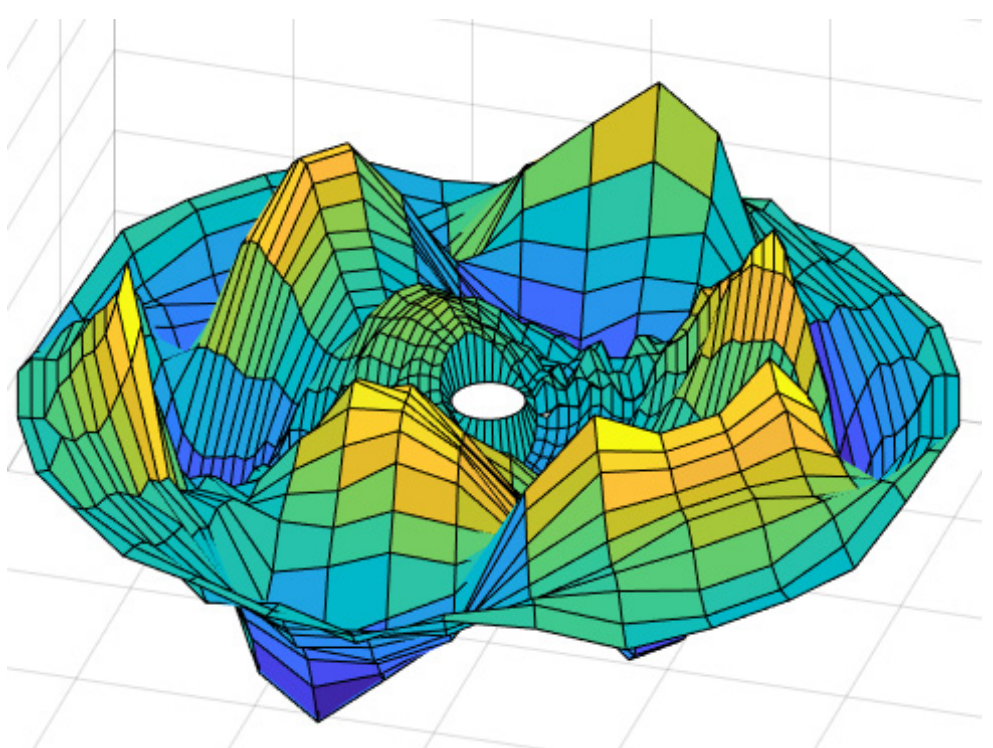

Figure 6. Time domain reflected $\mathrm{E}_{3}$ field at a given time instant. 


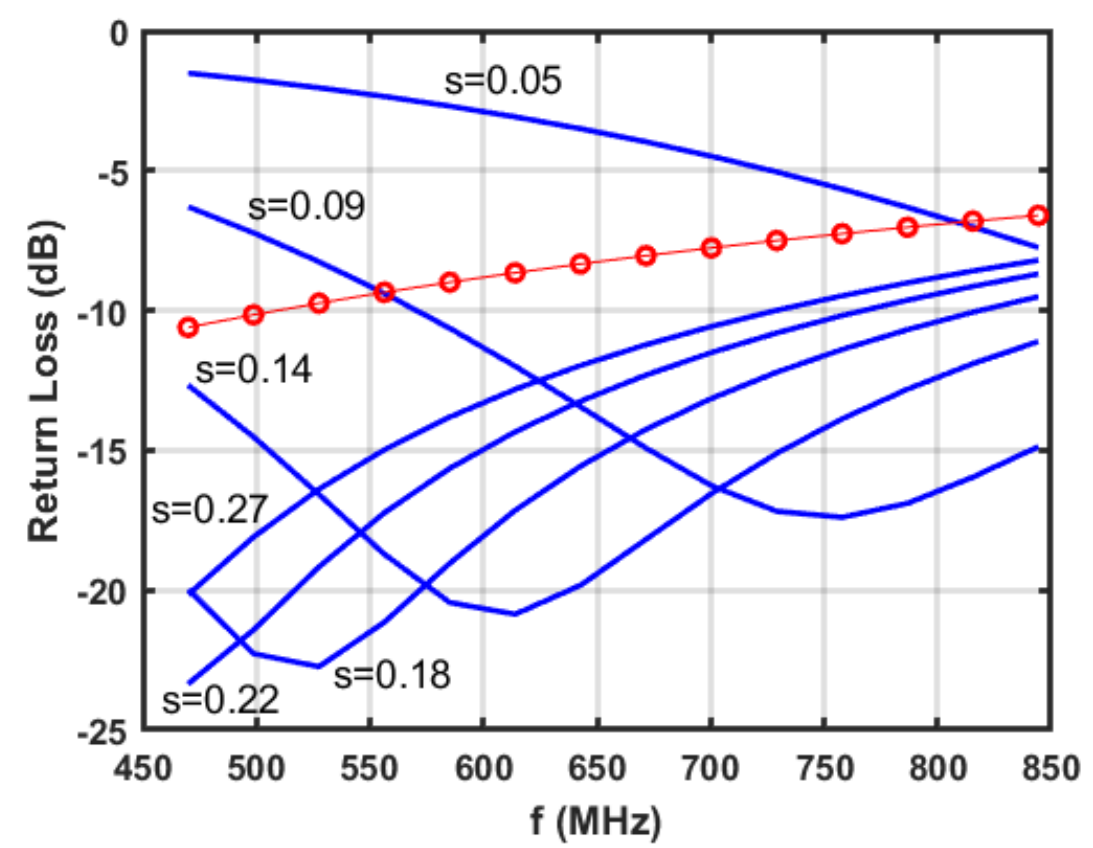

Figure 7. Return losses [470 MHz, $806 \mathrm{MHz}]$ ) in radome having wire $=0.8 \mathrm{~mm} \varnothing$, and thickness $=16 \mathrm{~mm}$ : (-o-)without helical wire. (-) with helical, wire spacing between turns $\mathrm{s}=0.05$, $0.9,0.14,0.18,0.22,0.27 \mathrm{~m}$.

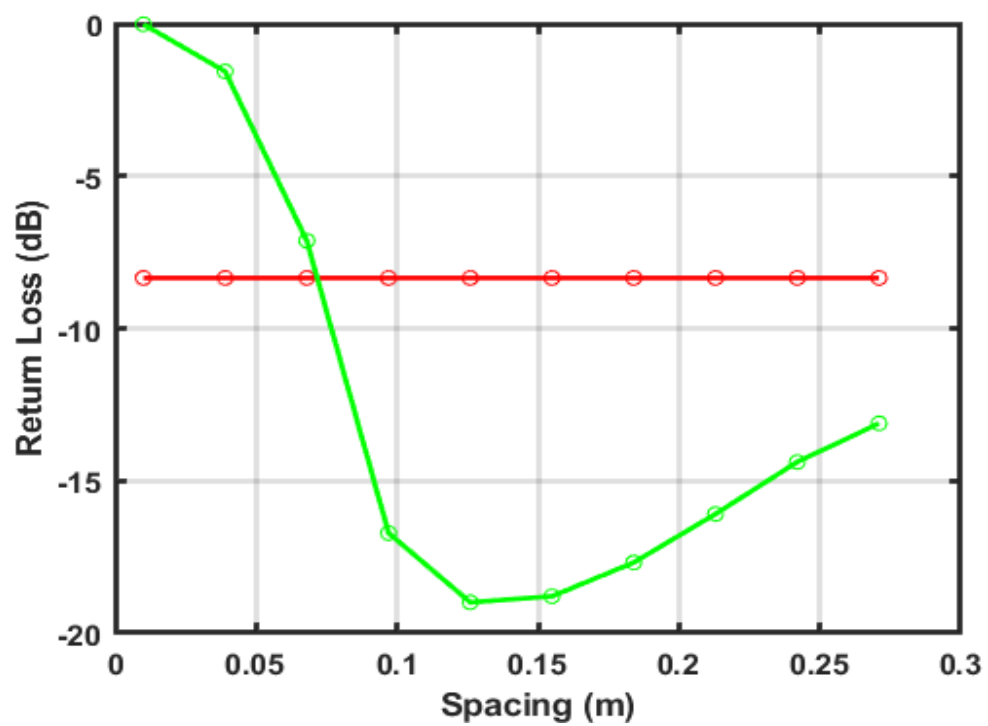

Figure 8. Average return losses in the frequency band [470 MHz, $806 \mathrm{MHz}$. Radome of wire $=0.8 \mathrm{~mm} \varnothing$, and thickness $=16 \mathrm{~mm}$ : (-o-) without helical wire. (-o-) with helical wire, spacing $\mathrm{s}=0.01-0.27 \mathrm{~m}$.

The program runs comfortably and fast on a pc with a NVIDIA GeForce GTX 970 GPU card, i7-8700 CPU at 3.2 GHz, and $32 \mathrm{~Gb}$ of RAM, windows 10, 64 bits.

The optimization scheme is set as follows. The GA is set to run 500 generations working with a population of 100 individuals:

Individual $_{i=1 \ldots 100}=(\text { Thickness }, \text { diameter }, \text { spacing })_{i=1 \ldots 100 i}=(00101111,01010101,11110000)_{i=1 \ldots 100}$

The probability of mutation is set $p m=0.8$, based in previous work and trials [42]; and the fitness function is set to the return losses as in (26) evaluated from the tangential fields.

Figure 9 shows the fitness versus generation. The fitness function as Equation (29) takes in account the average of transmission in the band [470 MHz, $806 \mathrm{MHz}$ ] as well 
as in the central frequency. By using this fitness there is convergence to $-24.32 \mathrm{~dB}$ after 130 generations. The obtained design parameters are:

- $\quad$ Thickness $=0.0100 \mathrm{~m}$

- $\quad$ Spacing $=0.1486 \mathrm{~m}$

- $\quad$ Wire diameter $=0.1 \mathrm{~mm}$

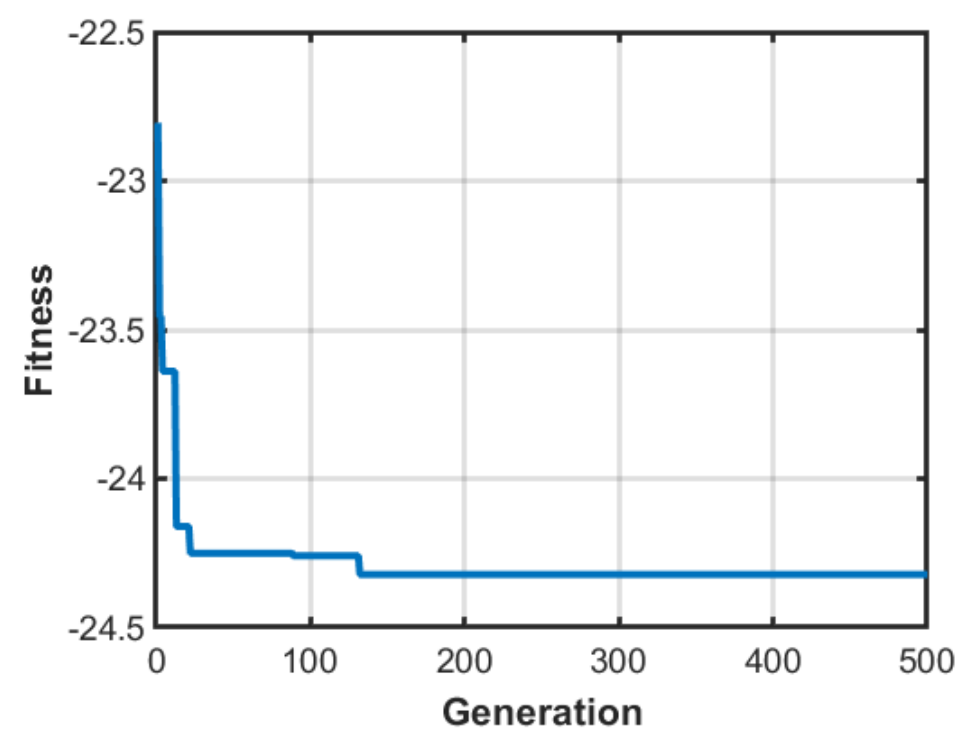

Figure 9. Fitness versus generation, fitness defined as the average return losses at the frequency band and central frequency as Equation (29).

The GA can be modified introducing changes in fitness, to analyze differences, for instance to take in account only the average transmission in frequency band [470 MHz, $806 \mathrm{MHz}$ ] with the fitness:

$$
\text { Fitness }_{i}=F\left(S 11_{i}\right)=\text { Average }(S 11[f 1, f 2])_{i}
$$

The GA achieves fitness $=-21.80 \mathrm{~dB}$, see Figure 10, providing the design parameters:

- $\quad$ Thickness $=0.0100 \mathrm{~m}$

- $\quad$ Spacing $=0.1565 \mathrm{~m}$

- $\quad$ Wire diameter $=0.1 \mathrm{~mm}$

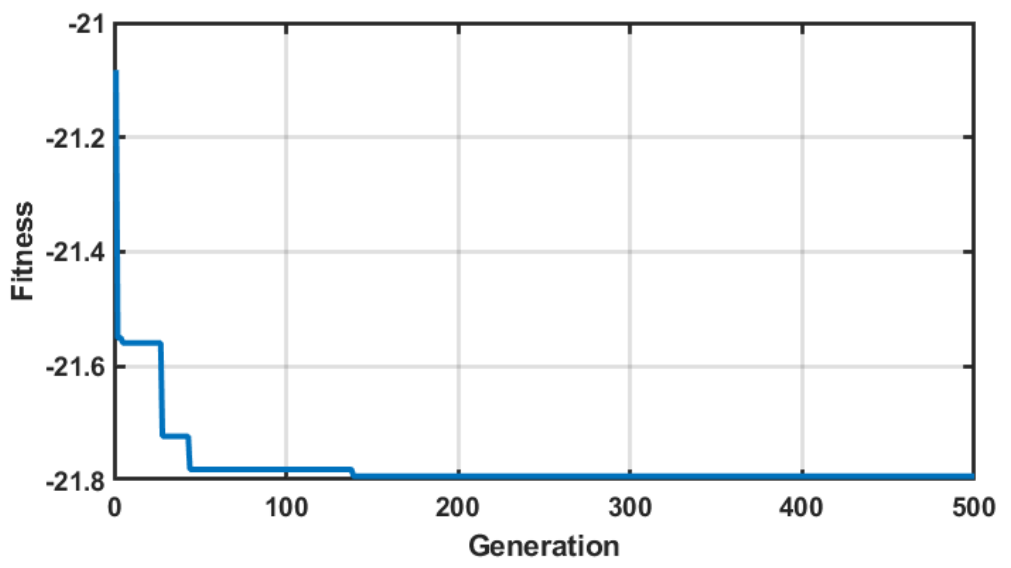

Figure 10. Fitness versus generation, fitness defined as the average reflection at the frequency band [470 MHz, $806 \mathrm{MHz}$ ], as Equation (30). 
However if we re-define the fitness as the best behavior at the central frequency:

$$
\text { Fitness }_{i}=F\left(S 11_{i}\right)=S 11\left(\frac{f 1+f 2}{2}\right)_{i}
$$

It achieves fitness $=-26.9 \mathrm{~dB}$, see Figure 11, and obtained design parameters are:

- $\quad$ Thickness $=0.0100 \mathrm{~m}$

- $\quad$ Spacing $=0.1502 \mathrm{~m}$

- $\quad$ Wire diameter $=0.1 \mathrm{~mm}$

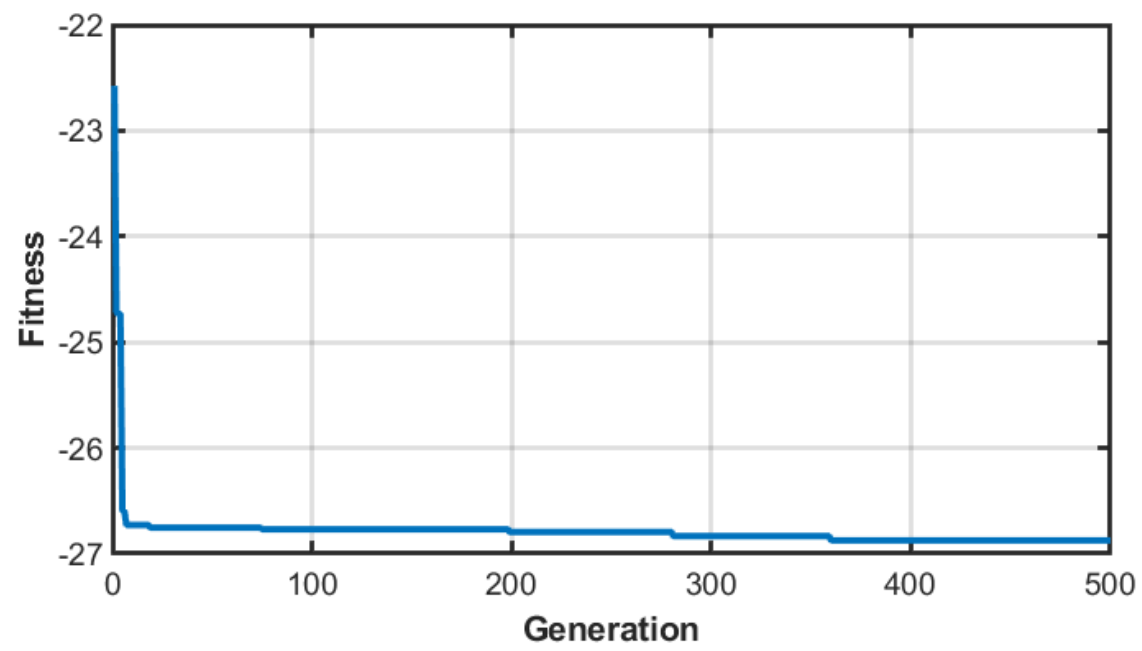

Figure 11. Fitness versus generation, fitness defined as the return losses at central frequency as Equation (31).

Convergence is observed to the same optimal values in any chosen fitness function. Results obtained with three different objective functions are shown in Table 1, and optimal parameters have negligible differences.

Table 1. Optimal parameters obtained through different objective functions.

\begin{tabular}{ccccc}
\hline Fitness & Reflection & $\begin{array}{c}\text { Thickness } \\
{[\mathbf{0 . 0 1}, \mathbf{0 . 0 5}] \mathbf{~ m}}\end{array}$ & $\begin{array}{c}\text { Spacing } \\
{[\mathbf{0 . 1 0 , 0 . 5 0 ] ~} \mathbf{~}}\end{array}$ & $\begin{array}{c}\text { Wire Diameter } \\
{[\mathbf{0 . 1}, \mathbf{1 . 0} \mathbf{~ m m}}\end{array}$ \\
\hline Equation (29) & $-24.32 \mathrm{~dB}$ & 0.01 & 0.1486 & 0.1 \\
Equation (30) & $-21.80 \mathrm{~dB}$ & 0.01 & 0.1565 & 0.1 \\
Equation (31) & $-26.9 \mathrm{~dB}$ & 0.01 & 0.1502 & 0.1 \\
\hline
\end{tabular}

Inside each population at each generation there is a large variability in fitness performance, as is shown in Figure 12, however there is a deep minimum for the fitness function when convergence is achieved. In the population of Figure 12 fitness oscillates between $-5 \mathrm{~dB}$ and $-27 \mathrm{~dB}$. Obviously we look for the deepest minimum that is $-27 \mathrm{~dB}$.

We observe a tendency of the optimization procedure to minimize the thickness of the dielectric material and the wire diameter. The spacing between turns of the helix is adapted to these parameters maintaining a value close to $0.15 \mathrm{~m}$.

If the boundaries are changed to increase the plastic cover thickness, for example in the interval $[0.025,0.05] \mathrm{m}$; the optimization chooses the smallest thickness. Therefore the limiting factor in the optimization is the dielectric thickness. Convergences with several bounds which are presented in Table 2 are shown in Figure 13. 


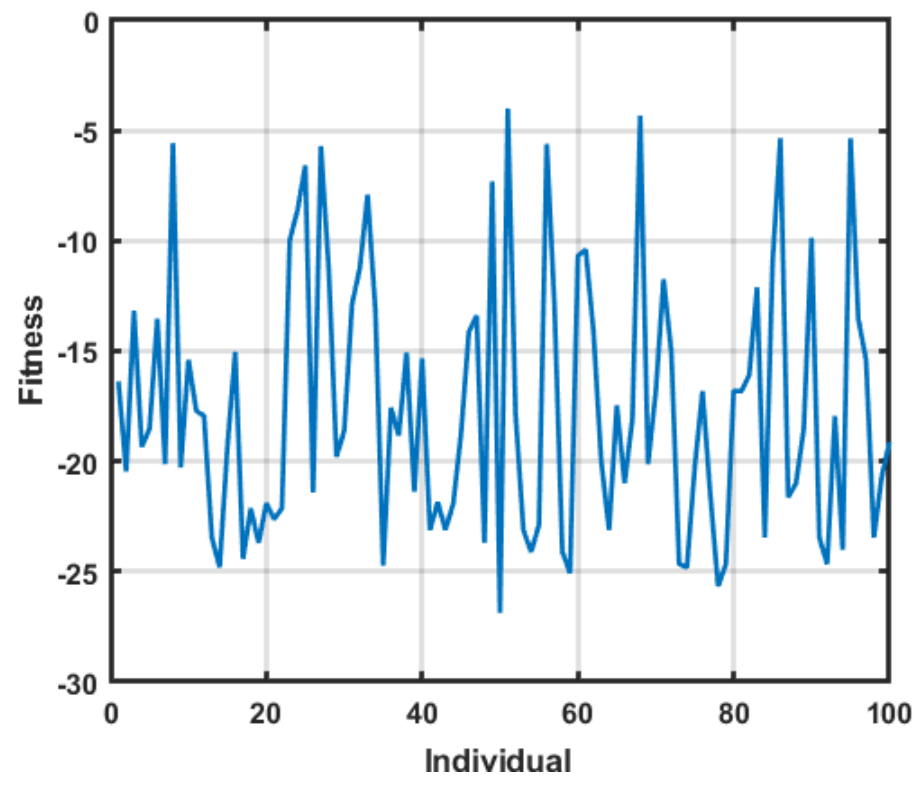

Figure 12. Fitness in the last best population, fitness defined as Equation (31).

Table 2. Optimal parameters obtained by looking for in different parameter bounds. Fitness as Equation (29).

\begin{tabular}{|c|c|c|c|c|}
\hline Bounds GA-Thickness, Spacing, Wire Ø (m) & Reflection & Thickness (m) & Spacing (m) & Wire $\varnothing(\mathrm{mm})$ \\
\hline (1) $[0.01,0.05] \times[0.10,0.50] \times\left[0.1 \times 10^{-9}, 1.0 \times 10^{-3}\right]$ & $-24.9 \mathrm{~dB}$ & 0.01 & 0.12 & 0.02 \\
\hline (2) $[0.14,0.20] \times[0.005,0.50] \times\left[0.8 \times 10^{-3}, 2.0 \times 10^{-3}\right]$ & $-7.4 \mathrm{~dB}$ & 0.14 & 0.11 & 1.0 \\
\hline (3) $[0.025,0.05] \times[0.01,0.50] \times\left[0.1 \times 10^{-9}, 1.0 \times 10^{-3}\right]$ & $-12.35 \mathrm{~dB}$ & 0.025 & 0.06 & 0.02 \\
\hline (4) $[0.05,0.10] \times[0.005,0.50] \times\left[0.1 \times 10^{-9}, 1.0 \times 10^{-3}\right]$ & $-11.74 \mathrm{~dB}$ & 0.100 & 0.29 & $1 \times 10^{-7}$ \\
\hline (5) $[0.05,0.10] \times[0.005,0.50] \times\left[1.0 \times 10^{-3}, 3.0 \times 10^{-3}\right]$ & $-10.34 \mathrm{~dB}$ & 0.100 & 0.50 & 1.00 \\
\hline (6) $[0.01,0.10] \times[0.005,0.50] \times\left[1.0 \times 10^{-3}, 3.0 \times 10^{-3}\right]$ & $-23.13 \mathrm{~dB}$ & 0.100 & 0.21 & 1.00 \\
\hline (7) $[0.01,0.10] \times[0.005,0.50] \times\left[1.0 \times 10^{-3}, 3.0 \times 10^{-3}\right]$ & $-23.54 \mathrm{~dB}$ & 0.008 & 0.25 & 1.00 \\
\hline
\end{tabular}

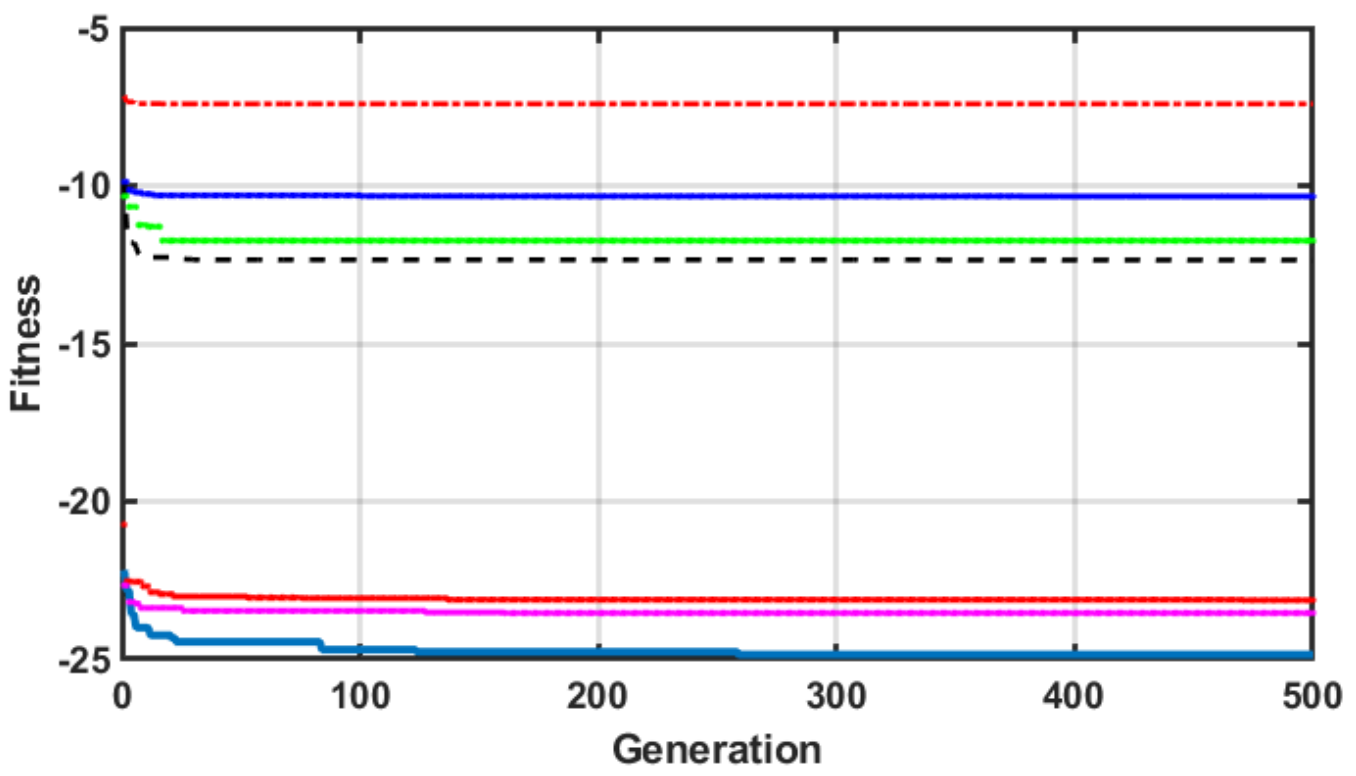

Figure 13. Fitness versus generation. Fitness defined in (29). Bounds in GA search of Table 2, cases (1)-(2)-(3)-(4)-(5)-(6)-(7) of Table 2. 


\subsection{Radiation Pattern}

The RACD system is designed specifically for UHF broadcast, and the manufacturing specifications are that the radome should minimally alter the original cross-dipole array pattern, especially outside the broadside direction. There is concern about the possibility of some degradation of the radiation pattern due to the presence of the helical wire. Although the helical wire improves the transmission, it works as a passive parasitic element which induced currents may influence the final radiation pattern of the whole RACD. The helical wire would operate as a monofilar helix antenna, and helix antenna may have two radiating operating modes: Axial mode and normal mode. Usually helix antenna operates in axial mode, in which it radiates along its axis circular polarization. This is the usual operating mode of the helix antenna for which it is designed. In normal mode the radiation is normal to the axis. From the original work of Kraus [52,53], we can guess that our design is correct. The normalized circumference of the helix is $C_{\lambda}=2 \pi \mathrm{r} / \lambda=10.7$, and the optimal normalized spacing $s_{\lambda}=s / \lambda$ is between $0.2-0.6$, therefore is out of the axial mode region, very far on top of the line $C_{\lambda}=2 \sqrt{ }\left(s_{\lambda}+1\right)$. The pitch angle $=\operatorname{atan}\left(s_{\lambda} / C_{\lambda}\right)$ is between $1^{\circ}-3^{\circ}$, therefore the absence of an axial mode is almost guaranteed. The helix works similarly as an array of circular loops, which are expected to radiate horizontal polarization in the direction normal to the axis. However we make some numerical calculations to confirm our hypothesis and analyze any undesired effect.

To analyze any far field pattern distortion we calculate the radiation pattern (RP) of the array with and without radome to analyze differences. Induced currents in the helical wire work as a helical antenna, thus re-radiating and may be distorting the far field of the array of crossed dipoles. The current in the helical wire can be calculated from the incident near field from the FDTD simulation. The tangential electric field induces superficial skin currents which depend on the wire diameter, conductivity and frequency. The near field is FDTD calculated at each point of the helical wire tangential to the wire, and the near to far field calculation [53] is carried out at $638 \mathrm{MHz}$ at the center of $470-806 \mathrm{MHz}$ band. Figure 14 shows the amplitude of induced current versus path along the discrete points of the helical wire using the optimum parameters of Table 2-(1). The current along the helix oscillates $\sim 10 \%$ and the number of oscillations is the nearest integer to the number of antennas of the RACD divided by three. The radiation of a RACD of 48 antennas is presented in Figure 15. The RACD is expected to be used in terrestrial broadcast radiating broadside, at $90^{\circ}$, horizontal polarized electric field (E-field), and parasitic radiation from currents induced in the helix may be a problem. In Figure 15 is shown the RP of the array without helix, the RP of helix induced currents and total RP. The shown RP's are H-plane, i.e., the vertical plane that contains the axis of the RACD that will be vertically mounted. The RP's start from $0^{\circ}$ in the end-fire direction (upside direction), end at $180^{\circ}$ in the ground direction. The RP of the array is symmetrical, and the RP of the helix is not, as array geometry is symmetrical in the horizontal plane, and the helix is not. Although the current has a symmetrical distribution along the wire, the position of each small, discrete current, along the wire is not symmetrical. The helix in the radome covers the whole array starting at $\lambda / 2$ below the first antenna and ending $\lambda / 2$ on top of the last antenna. The helix RP has three lobes, the "main lobe" at $90^{\circ}$, but also has two lobes $1.3 \mathrm{~dB}$ higher than the main lobe at $35^{\circ}$ and $145^{\circ}$ having a width of $8^{\circ}$ from maxima. The helix has no axial mode [48], as we expected, but has three radiating lobes. The results of Figure 15 are normalized to the maximum radiated power, and although the helix radiates two undesired lobes, the power of them is $7.8 \mathrm{~dB}$ below the power radiated by the array, and its contribution to the total RP is low, therefore RP distortion is negligible. The total RP (array + helix) is shown in blue color in Figure 15, the total RP overlaps with the desired RP from the array (in green color) having a negligible deviation from the desired RP at $35^{\circ}$ and $145^{\circ}$. 


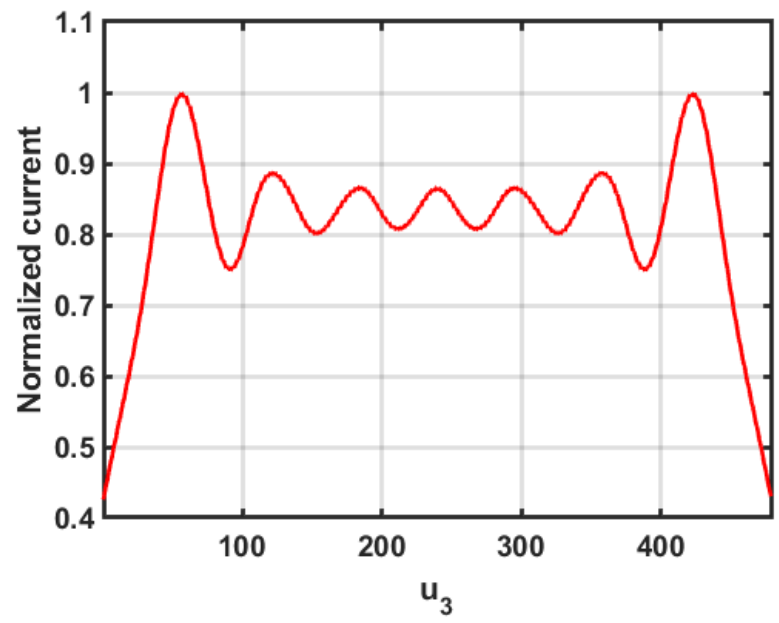

(a)

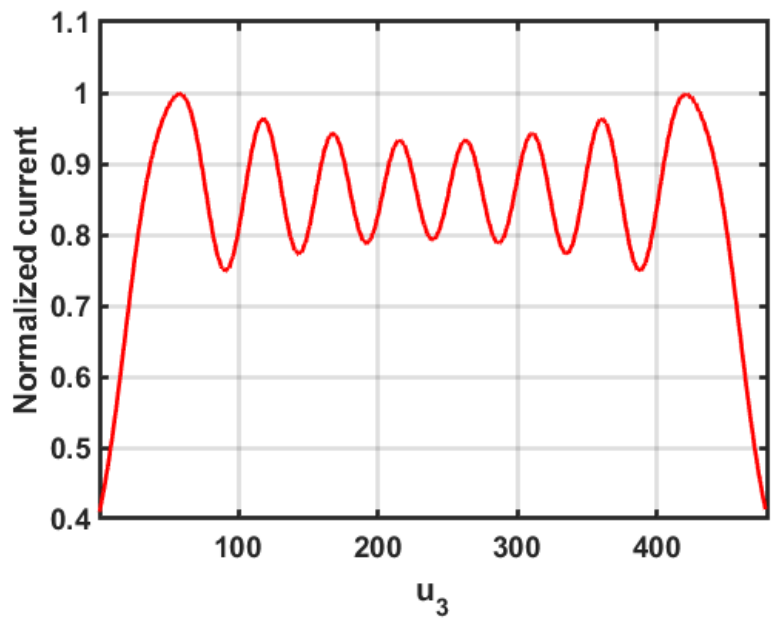

(b)

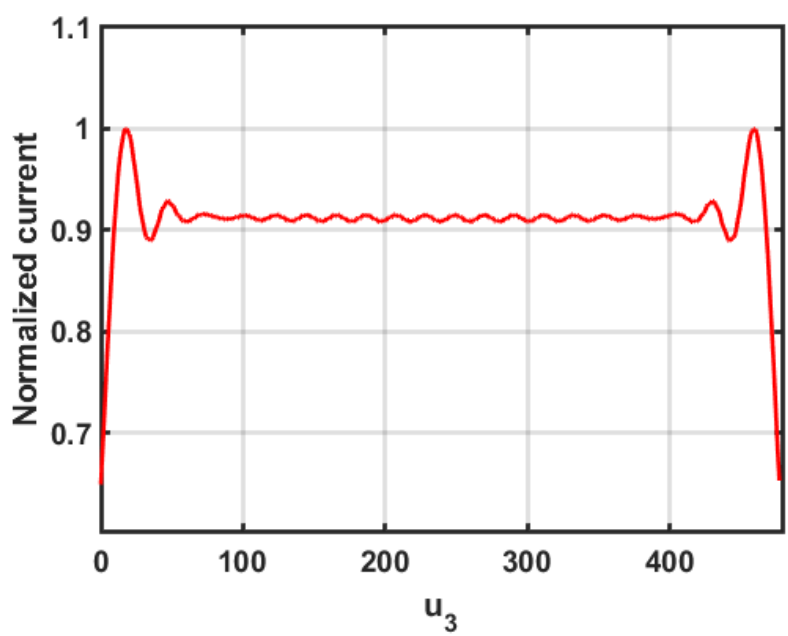

(c)

Figure 14. Induced current in discretized points of helix by near tangential electric field. (a) $n=20$ antennas. (b) $n=21$ antennas. (c) $n=48$ antennas. 


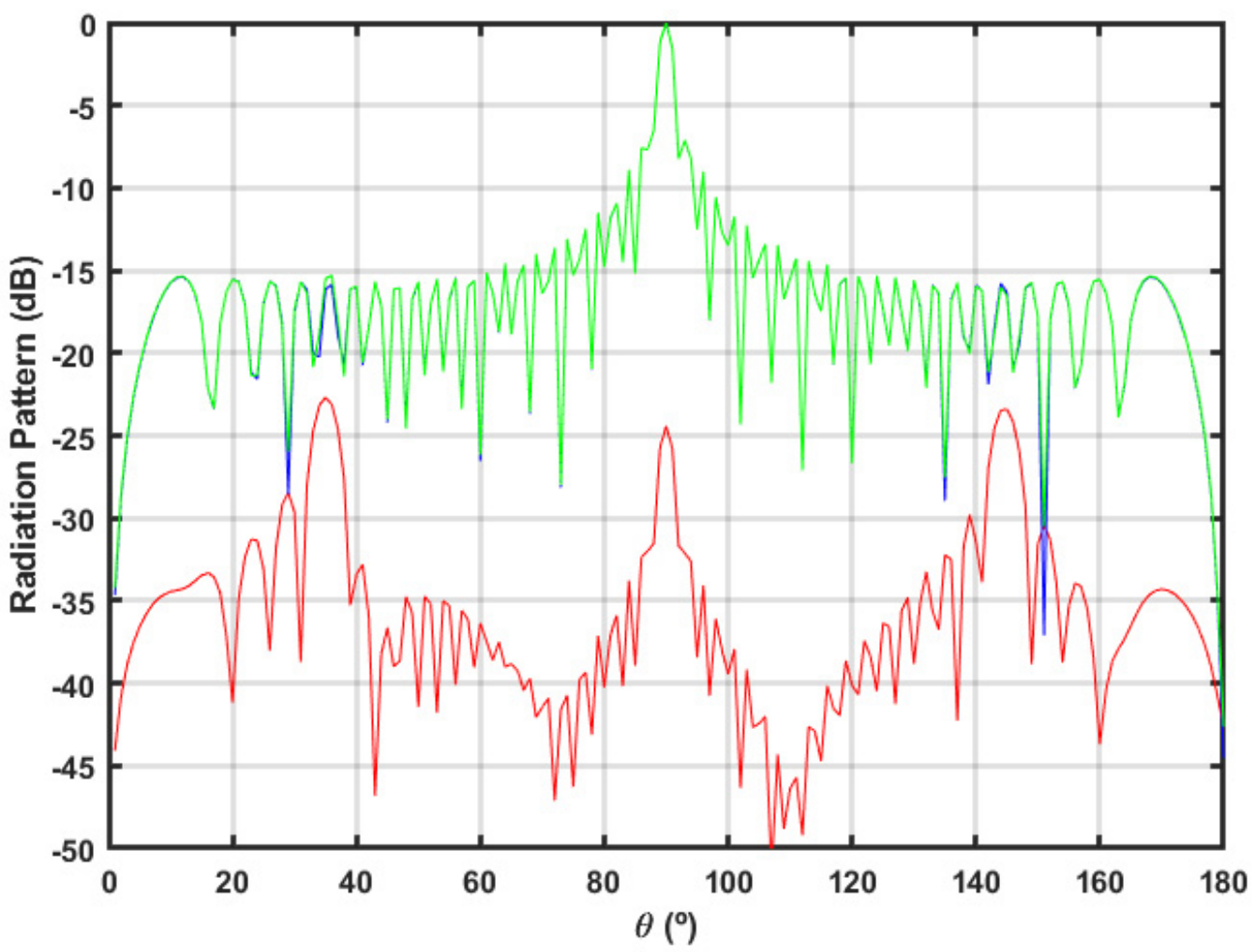

Figure 15. Radiation Pattern (RP) of RACD with 48 antennas case Table 2-(1) step $=0.12 \mathrm{~m}$ : $(-) \mathrm{RP}$ without helix. (-) Parasitic RP of helix. (-) Total RP.

Finally, the RACD is intended to operate horizontally polarized E-field at $90^{\circ}$, that is, the field originally radiated by the array of crossed dipoles at $90^{\circ}$. In Figure 16 are presented the RP of horizontal polarized field $\left(\mathrm{E}_{\varphi}\right)$, and polarized field $\mathrm{E}_{\theta}$ with and without radome. The helix has a clear influence on polarization. This is due to the vertical component of the current, due to the vertical projection of the helical wire. The RACD does not radiate pure horizontal polarization, however at $\pm 7^{\circ}$, from the maximum the vertical polarization is $10 \mathrm{~dB}$ below the horizontal polarization, at $90^{\circ}$ is $30 \mathrm{~dB}$ down, for the intended purpose of the RACD the improvement in return losses that provides the helical wire outperforms the losses in power by undesired cross polarization.

The results for a RACD of 20 antennas are also presented to provide a better graphical view of other aspects of the RACD performance, because the number of lobes increases with the number of antennas, making visual inspection of the plots difficult. We simulate the RACD with 20 antennas to provide a clear view of the influence of the helical wire in the radiation pattern, also to clearly see the main lobes and nulls for interpretation. The radome starts at $\lambda / 2$ below the first antenna and ends $\lambda / 2$ on top of the last antenna for any number of antennas, therefore depending on the number of antennas we have a different height and volume of the whole structure. Results are shown in Figures 17 and 18. The number of antennas changes the RP's. A smaller number of antennas lead to lower directivity. However, where it is noticeable is especially in the RP of the helix, where four secondary lobes arise in a pattern without symmetry. However, the levels of these lobes although relatively high within the helix RP have a very low power with respect to what the dipoles radiate. The effect of the helix is negligible, on the order of $20 \mathrm{~dB}$ below the dipole radiation, which is why in Figure 16 the green line is superimposed on the blue line, and blue line is not visible. The RACD of 20 antennas does not radiate horizontal polarization, with exception of $90^{\circ}$, as in previous case, however at $\pm 7^{\circ}$, from the maximum the vertical polarization is $10 \mathrm{~dB}$ below the horizontal polarization, at $90^{\circ}$ is below $30 \mathrm{~dB}$. 


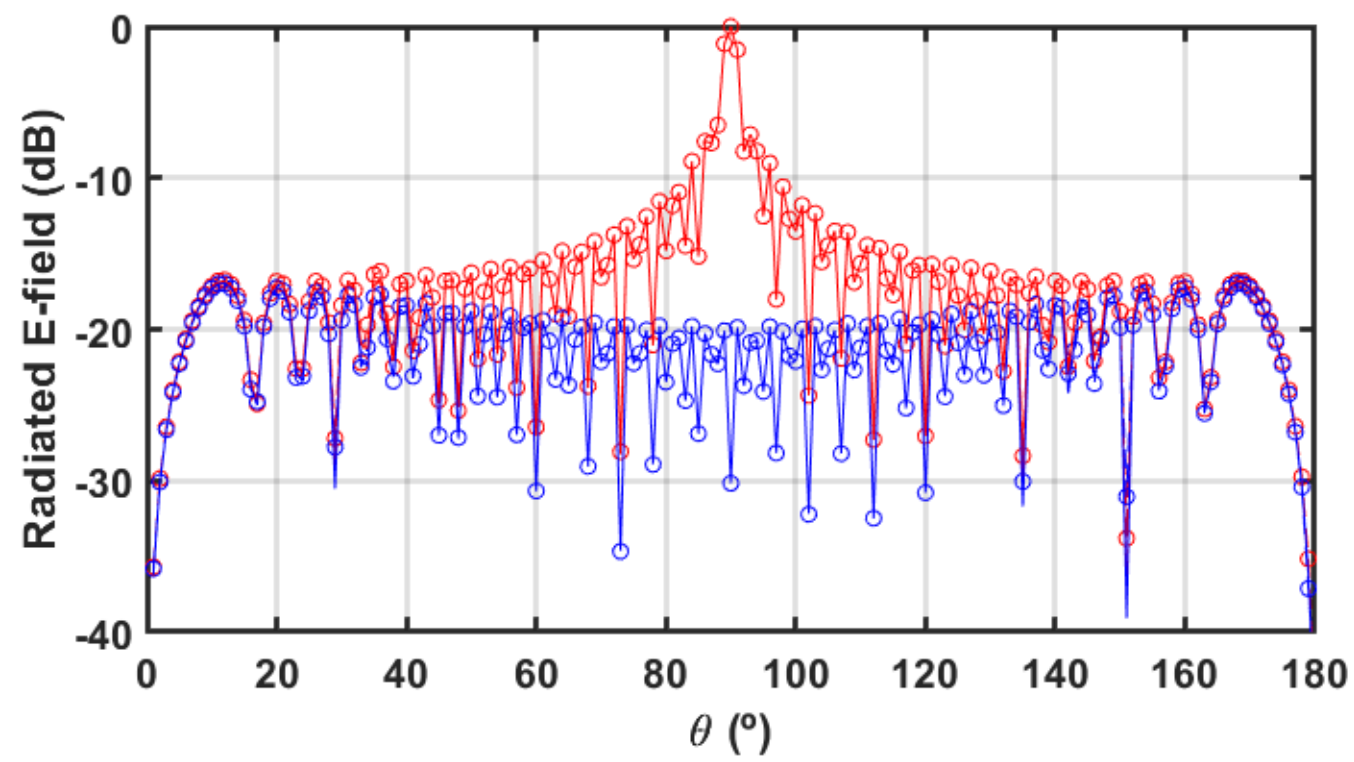

Figure 16. Radiated E-fields. Case Table 2-(1) with 48 antennas step $=0.12 \mathrm{~m}$ : (一) Horizontal polarization $\left(\mathrm{E}_{\varphi}\right)$. (-o-) Horizontal polarization $\left(\mathrm{E}_{\varphi}\right)$ with helix. (-) $\mathrm{E}_{\theta}$ without helix. (-o-) $\mathrm{E}_{\theta}$ with helix.

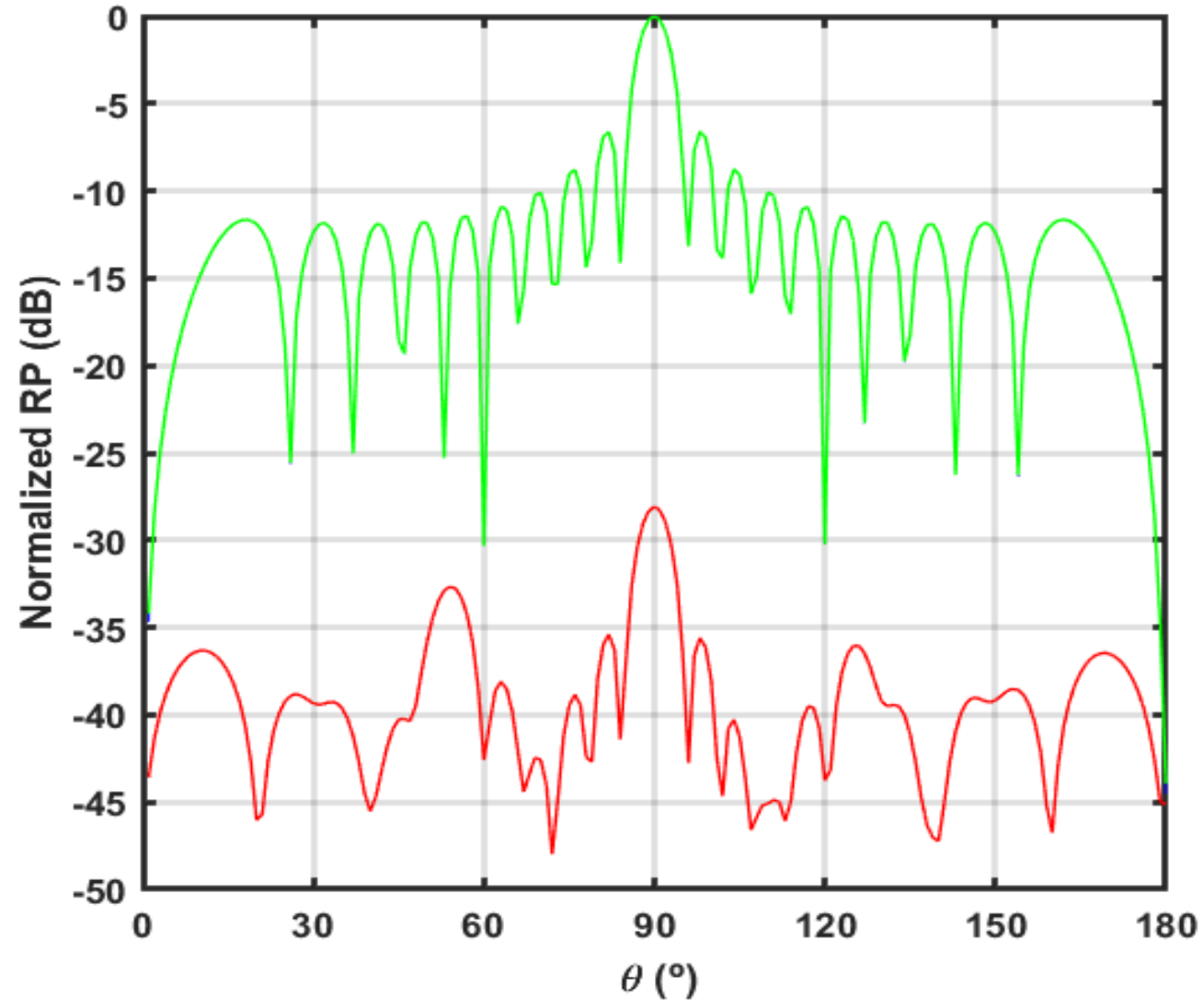

Figure 17. Radiation Pattern (RP) of RACD with 20 antennas case Table 2-(1) step $=0.12 \mathrm{~m}:(-) \mathrm{RP}$ without helix. (-) Parasitic RP of helix. (-) Total RP. 


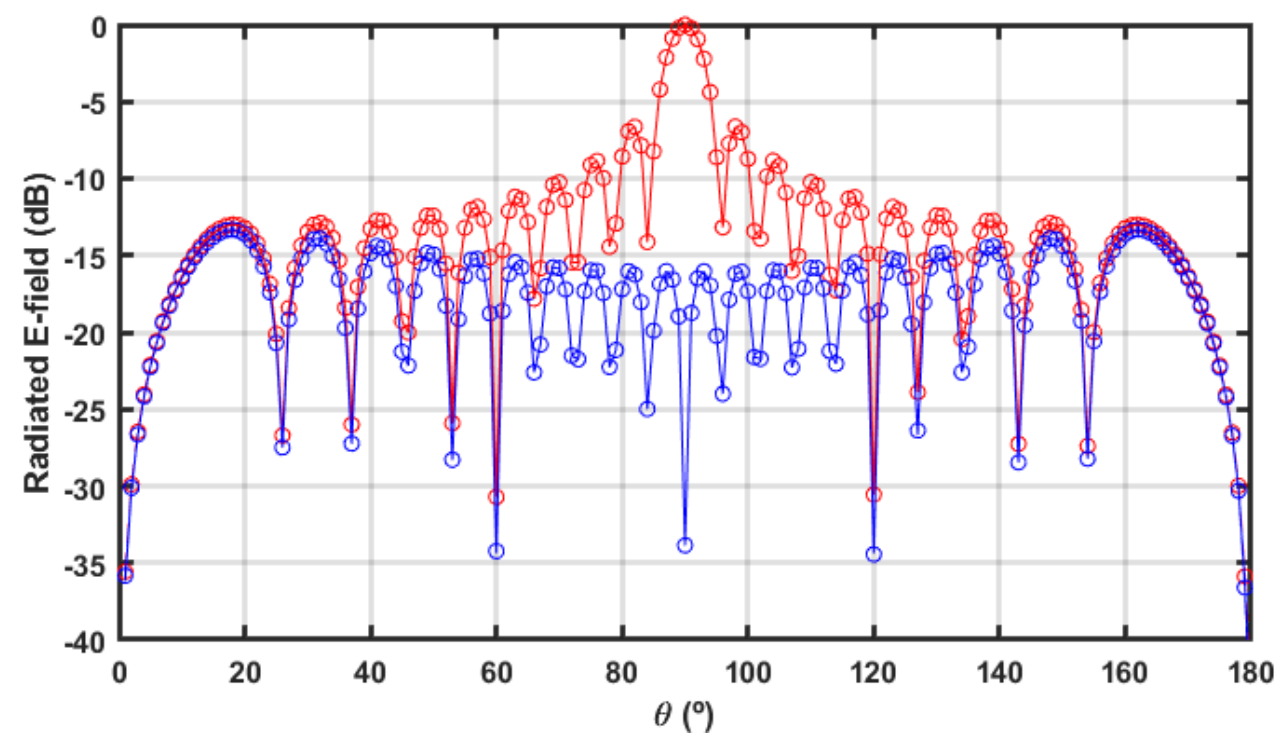

Figure 18. Radiated E-fields. Case Table 2-(1) with 20 antennas step $=0.12 \mathrm{~m}$ : (-) Horizontal polarization $\left(\mathrm{E}_{\varphi}\right)$. (-o-) Horizontal polarization $\left(\mathrm{E}_{\varphi}\right)$ with helix. (-) $\mathrm{E}_{\theta}$ without helix. (-o-) $\mathrm{E}_{\theta}$ with helix.

If we analyze the Table 2, depending on the bounds, the GA may converge to unacceptable return losses. This is the case of Table 2-(2), return losses perform worse. The results of introducing the helix worsen the performance of the radome, its introduction does not have any advantage with respect to the only-dielectric radome. This bad performance also appears in the RP's and polarization, shown in Figures 19 and 20. The helix has also a stronger contribution to the total RP (see Figure 19), changing the null distribution, but also increasing $10 \mathrm{~dB}$ the radiated power to $0^{\circ}$ and $180^{\circ}$. Polarization is shown in Figure 20, the results in circles plot the polarization without helix, and the solid lines with helix. The results for horizontal polarization $\mathrm{E}_{2}=\mathrm{E}_{\varphi}$, overlap in the interval $60-120^{\circ}$, with negligible differences out of this. The $\mathrm{E}_{\theta}$ polarization has slightly more differences, in the interval $50-70^{\circ}$ that increase to almost $5 \mathrm{~dB}$ near $0^{\circ}$ and $180^{\circ}$, to ground and zenith of the RACD.

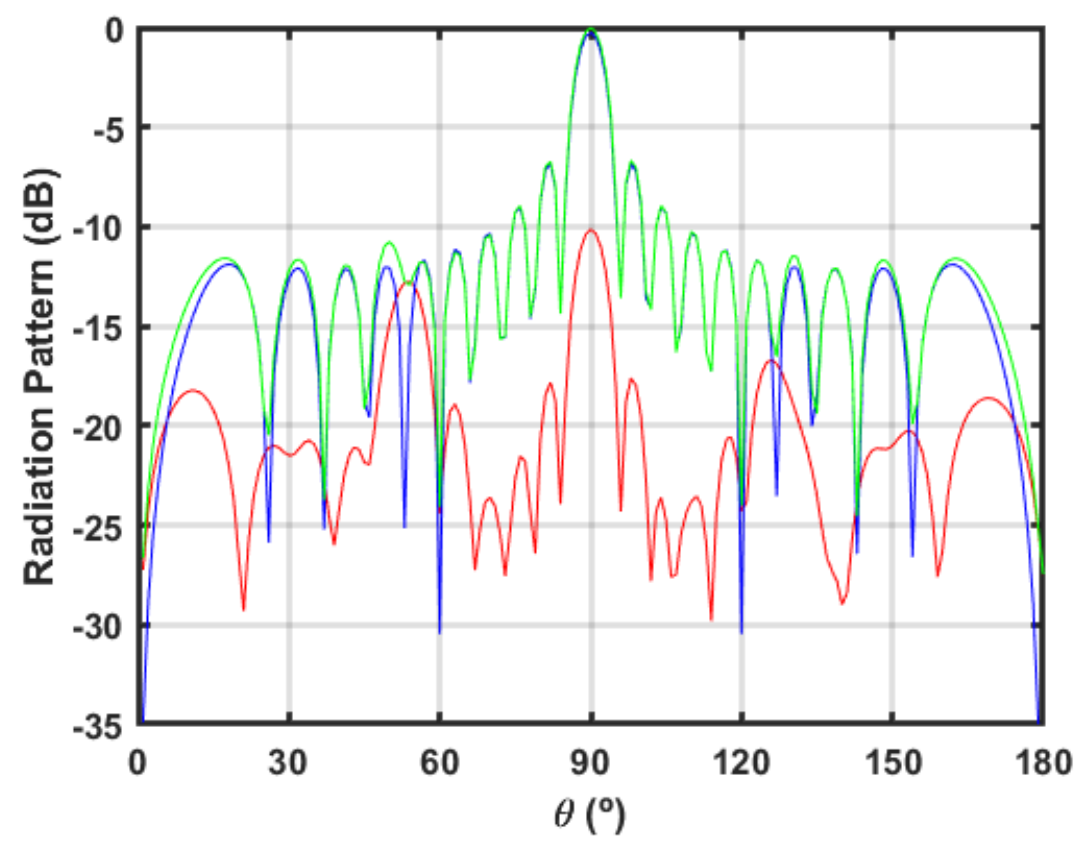

Figure 19. Radiation Pattern (RP) of RACD with 20 antennas case Table 2-(2) step $=0.11 \mathrm{~m}$ : (一) RP without helix. (一) Parasitic RP of helix. (一) Total RP. 


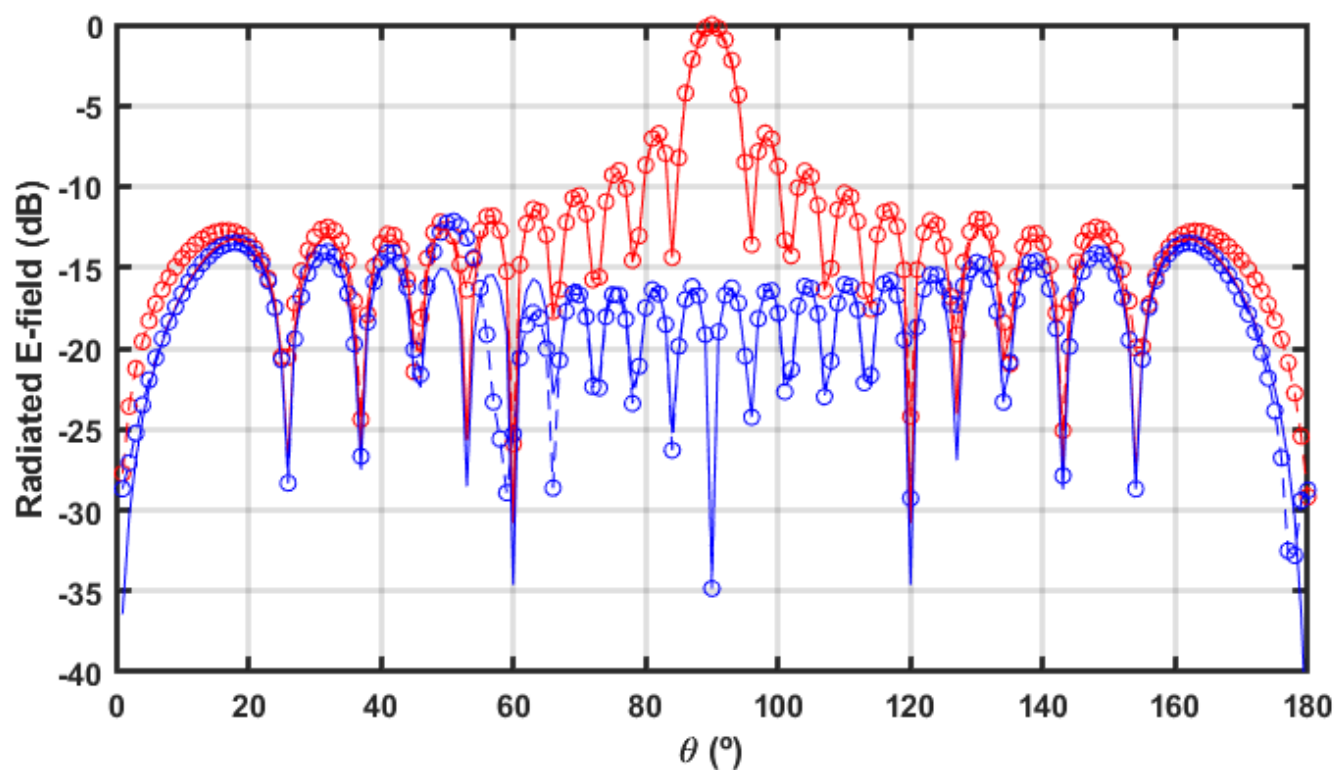

Figure 20. Radiated E-field. Case Table 2-(2) with 20 antennas step $=0.11 \mathrm{~m}$ : (一) Horizontal polarization $\left(E_{\varphi}\right)$. (-o-) Horizontal polarization $\left(E_{\varphi}\right)$ with helix. $(-) E_{\theta}$ without helix. (-o-) $E_{\theta}$ with helix.

The helix increases $\mathrm{E}_{3}=\mathrm{E}_{\theta}$ polarization, this is due to the current induced along the axial direction in the helix. However, in every case, in a beam of 14 degrees wide the cross polarization is $10 \mathrm{~dB}$ down, also, in a very narrow beam of a few degrees in broadside direction this level is below $30 \mathrm{~dB}$, i.e., at broadside direction the horizontal polarization is kept.

Obviously, the helix has influence on the radiation pattern of RACD, although this influence is negligible for near broadside radiation, and is outweighed by the benefit of better matching of around $10 \mathrm{~dB}$ in the whole frequency band. However, a careful analysis is necessary to avoid the appearing of undesired side lobes that may influence total performance.

\section{Conclusions}

In this work we address a complex electromagnetic problem, the design and optimization of a large radome to protect a large array of crossed dipoles. The FDTD numerical technique using general curvilinear coordinates seems appropriated when combined with a search optimization algorithm, as it is the genetic algorithm. We use sub-cell features inside the curvilinear FDTD to give proper modeling of thin dielectrics and thin wires. Numerical FDTD runs inside GA are feasible using parallelization in GPU. A helical wire is used to give a better matching to the dielectric cover. The proposed structure to cover the array of crossed dipoles is technically feasible by improving transmission around $10 \mathrm{~dB}$, however, this introduces low levels of cross polarization, also undesired side lobes, depending on the parameters. Further analysis shows that for the intended use of the RACD system, which is to provide high gain at broadside direction with horizontal polarization, these are minor effects. The benefit of introducing a helix outweighs the detriments of cross-polarization and side lobes by doing a rigorous analysis and striving for an optimized design.

Author Contributions: Conceptualization, E.A.N. and J.A.P.; methodology, E.A.N.; software, E.N.M.; validation, E.N.-M., A.S.; formal analysis, E.A.N.; investigation, J.A.P.; resources, E.N.-M.; data curation, A.S.; writing-original draft preparation, J.A.P.; writing-review and editing, E.A.N.; visualization, E.N.-M.; project administration, J.F. and S.T.-R.; funding acquisition, J.F. and S.T.-R. All authors have read and agreed to the published version of the manuscript.

Funding: This research was funded by the government of Spain, MINECO, grant number FIS201790102-R. 
Acknowledgments: In memory of Vicente Such from University of València who started this complex work, and Juan Antonio Morente-Chiquero, from University of Granada, one of the most outstanding experts in computational electromagnetics.

Conflicts of Interest: The authors declare no conflict of interest.

\section{References}

1. Gimeno, B.; Such, V.; Garcia, A.C.; Cruz, J.L.; Navarro, E. Transmission-line model to analyze a multistage polarizer rotator. Microw. Opt. Technol. Lett. 1991, 4, 113-117. [CrossRef]

2. Gimeno, B.; Cruz, J.; Navarro, E.; Such, V. Electromagnetic Scattering by a Strip Grating with Plane-Wave Three-Dimensional Oblique Incidence by Means of Decomposition into E-Type and H-Type Modes. J. Electromagn. Waves Appl. 1993, 7, 1201-1219. [CrossRef]

3. Waqas, H.M.; Shi, D.; Imran, M.; Khan, S.Z.; Tong, L.; E Ahad, F.; Zaidi, A.A.; Iqbal, J.; Ahmed, W. Conceptual Design of Composite Sandwich Structure Submarine Radome. Materials 2019, 12, 1966. [CrossRef] [PubMed]

4. $\quad$ Einziger, P.D.; Felsen, L. Ray analysis of two-dimensional radomes. IEEE Trans. Antenn. Propag. 1983, 31, 870-884. [CrossRef]

5. Li, H.-Y.; Li, C.-M.; Gao, J.-G.; Sun, W.-F. Ameliorated Mechanical and Dielectric Properties of Heat-Resistant Radome Cyanate Composites. Molecules 2020, 25, 3117. [CrossRef]

6. Friederich, F.; May, K.H.; Baccouche, B.; Matheis, C.; Bauer, M.; Jonuscheit, J.; Moor, M.; Denman, D.; Bramble, J.; Savage, N. Terahertz Radome Inspection. Photonics 2018, 5, 1. [CrossRef]

7. Kim, J.; Lee, S.; Shin, H.; Jung, K.-Y.; Choo, H.; Park, Y.B. Radiation from a Cavity-Backed Circular Aperture Array Antenna Enclosed by an FSS Radome. Appl. Sci. 2018, 8, 234. [CrossRef]

8. Rogers Corporation. High Frequency Circuit Material Data Sheets. Available online: http://www.rogerscorp.com/acs/literature aspx (accessed on 5 August 2021).

9. Duan, Z.; Abomakhleb, G.; Lu, G. Perforated Medium Applied in Frequency Selective Surfaces and Curved Antenna Radome. Appl. Sci. 2019, 9, 1081. [CrossRef]

10. Hessel, A.; Sureau, J.-C. Resonances in circular arrays with dielectric sheet covers. IRE Trans. Antennas Propag. 1973, 21, 159-164. [CrossRef]

11. Montgomery, J. Scattering by an infinite periodic array of thin conductors on a dielectric sheet. IRE Trans. Antennas Propag. 1975, 23, 70-75. [CrossRef]

12. Pelton, E.; Munk, B. Scattering from periodic arrays of crossed dipoles. IRE Trans. Antennas Propag. 1979, 27, 323-330. [CrossRef]

13. Cwik, T.; Mittra, R. Scattering from a periodic array of free-standing arbitrarily shaped perfectly conducting or resistive patches. IRE Trans. Antennas Propag. 1987, 35, 1226-1234. [CrossRef]

14. Monni, S.; Gerini, G.; Neto, A.; Tijhuis, A.G. Multimode Equivalent Networks for the Design and Analysis of Frequency Selective Surfaces. IEEE Trans. Antennas Propag. 2007, 55, 2824-2835. [CrossRef]

15. Paris, D.T. Computer Aided Radome Analysis. IEEE Trans. Antennas Propag. 1970, 18, 7-15. [CrossRef]

16. Navarro, E.; Segura, J.; Soriano, A.; Such, V. Modeling of Thin Curved Sheets with the Curvilinear FDTD. IEEE Trans. Antennas Propag. 2004, 52, 342-346. [CrossRef]

17. You, J.W.; Tan, S.R.; Zhou, X.Y.; Yu, W.M.; Cui, T.J. A New Method to Analyze Broadband Antenna-Radome Interactions in Time-Domain. IEEE Trans. Antennas Propag. 2013, 62, 334-344. [CrossRef]

18. Shlager, K.L.; Schneider, J.B. A selective survey of the Finite-Difference Time-Domain Literature. IEEE Antennas Propag. Mag. 1995, 37, 39-56. [CrossRef]

19. Navarro, E.; Gimena, B.; Cruz, J. Modelling of periodic structures using the finite difference time domain method combined with the Floquet theorem. Electron. Lett. 1993, 29, 446-447. [CrossRef]

20. Navarro, E.; Bordallo, T.; Navasquillo-Miralles, J. FDTD characterization of evanescent modes-multimode analysis of waveguide discontinuities. IEEE Trans. Microw. Theory Tech. 2000, 48, 606-610. [CrossRef]

21. Navarro, E.; Such, V.; Gimeno, B.; Cruz, J.L. T-junctions in square coaxial waveguide: A FD-TD approach. IEEE Trans. Microw. Theory Tech. 1994, 42, 347-350. [CrossRef]

22. Navarro, E.A.; Such, V.; Gimeno, B.; Cruz, J.L. Analysis of H-plane waveguide discontinuities with an improved FD-TD algorithm. IEE Proc.-H 1992, 139, 183-185. [CrossRef]

23. Reig, C.; Navarro, E.A.; Such, V. Full-wave FDTD design and analysis of wideband microstrip-to-waveguide transitions. Microw. Opt. Technol. Lett. 2003, 38, 317-320. [CrossRef]

24. Navarro, E.; Sangary, N.; Wu, C.; Litva, J. Analysis of a coupled patch antenna with application in personal communications. IEE Proc. Microwaves Antennas Propag. 1995, 142, 495-497. [CrossRef]

25. Reig, C.; Navarro, E.A.; Such, V. FDTD analysis of an E-sectoral horn excited by an opened microstrip. Microw. Opt. Technol. Lett. 1996, 13, 294-297. [CrossRef]

26. Wu, C.; Navarro, E.A.; Navasquillo, J.; Litva, J. FDTD signal extrapolation using a finite impulse response neural network model. Microw. Opt. Technol. Lett. 1999, 21, 325-330. [CrossRef]

27. Wu, C.; Navarro, A.; Litva, J. Combination of finite impulse response neural network technique with FDTD method for simulation of electromagnetic problems. Electron. Lett. 1996, 32, 1112-1113. [CrossRef] 
28. Navarro, E.; Gallart, L.; Cruz, J.; Gimeno, B.; Such, V. Accurate absorbing boundary conditions for the FDTD analysis of H-plane waveguide discontinuities. IEE Proc. Microwaves Antennas Propag. 1994, 141, 59-61. [CrossRef]

29. Wu, C.; Navarro, E.A.; Chung, P.Y.; Litva, J. Modeling of waveguide structures using the nonorthogonal FDTD method with a PML absorbing boundary. Microw. Opt. Technol. Lett. 1995, 8, 226-228. [CrossRef]

30. Maloney, J.; Smith, G. The use of surface impedance concepts in the finite-difference time-domain method. IEEE Trans. Antennas Propag. 1992, 40, 38-48. [CrossRef]

31. Maloney, J.; Smith, G. The efficient modeling of thin material sheets in the finite-difference time-domain (FDTD) method. IEEE Trans. Antennas Propag. 1992, 40,323-330. [CrossRef]

32. Maloney, J.; Smith, G. A comparison of methods for modeling electrically thin dielectric and conducting sheets in the finitedifference time-domain (FDTD) method. IEEE Trans. Antennas Propag. 1993, 41, 690-694. [CrossRef]

33. Stratton, J.A. Electromagnetic Theory; McGray Hill: New York, NY, USA, 1941.

34. Holland, R. Finite-Difference Solution of Maxwell's Equations in Generalized Nonorthogonal Coordinates. IEEE Trans. Nucl. Sci. 1983, 30, 4589-4591. [CrossRef]

35. Ta, S.X.; Park, I.; Ziolkowski, R. Crossed Dipole Antennas: A review. IEEE Antennas Propag. Mag. 2015, 57, 107-122. [CrossRef]

36. Calatayud, R.; Navarro-Modesto, E.; Navarro-Camba, E.A.; Sangary, N.T. Nvidia CUDA parallel processing of large FDTD meshes in a desktop computer: FDTD-Matlab on GPU. In Proceedings of the EATIS 2020, Aveiro, Portugal, 25-27 November 2020; pp. 3810-4193. [CrossRef]

37. Weile, D.; Michielssen, E. Genetic algorithm optimization applied to electromagnetics: A review. IEEE Trans. Antennas Propag. 1997, 45, 343-353. [CrossRef]

38. Elias, I.; Rubio, J.D.J.; Martinez, J.P.; Vargas, T.M.; Garcia, V.; Mujica-Vargas, D.; Meda-Campaña, J.A.; Pacheco, J.; Gutierrez, G.J.; Zacarias, A. Genetic Algorithm with Radial Basis Mapping Network for the Electricity Consumption Modeling. Appl. Sci. 2020, 10, 4239. [CrossRef]

39. Park, K.M.; Shin, D.; Chi, S.D. Variable Chromosome Genetic Algorithm for Structure Learning in Neural Networks to Imitate Human Brain. Appl. Sci. 2019, 9, 3176. [CrossRef]

40. Xin, J.; Zhong, J.; Yang, F.; Cui, Y.; Sheng, J. An Improved Genetic Algorithm for Path-Planning of Unmanned Surface Vehicle. Sensors 2019, 19, 2640. [CrossRef]

41. Marcano, D.; Duran, F. Synthesis of antenna arrays using genetic algorithms. IEEE Antennas Propag. Mag. 2000, 42, 12-20. [CrossRef]

42. Fornieles-Callejón, J.; Salinas, A.; Redondo, S.T.; Portí, J.; Méndez, A.; Navarro, E.A.; Morente-Molinera, J.A.; Soto-Aranaz, C.; Ortega-Cayuela, J.S. Extremely low frequency band station for natural electromagnetic noise measurement. Radio Sci. 2014, 50, 191-201. [CrossRef]

43. Ansys HFSS. Broadband Adaptive Meshing. Available online: https://www.ansys.com/content/dam/product/electronics/ hfss / ab-ansys-hfss-adaptive-broadband-meshing.pdf (accessed on 2 September 2021).

44. Martinez, P.A.; Navarro, E.A.; Victoria, J.; Suarez, A.; Torres, J.; Alcarria, A.; Perez, J.; Amaro, A.; Menendez, A.; Soret, J. Design and Study of a Wide-Band Printed Circuit Board Near-Field Probe. Electronics 2021, 10, 2201. [CrossRef]

45. Porti, J.; Morente, J.; Khalladi, M.; Gallego, A. Comparison of thin-wire models for TLM method. Electron. Lett. 1992, 28, 1910-1911. [CrossRef]

46. Taniguchi, Y.; Baba, Y.; Nagaoka, N.; Ametani, A. An Improved Thin Wire Representation for FDTD Computations. IEEE Trans. Antennas Propag. 2008, 56, 3248-3252. [CrossRef]

47. Asada, T.; Baba, Y.; Nagaoka, N.; Ametani, A. An Improved Thin Wire Representation for FDTD Transient Simulations. IEEE Trans. Electromagn. Compat. 2014, 57, 1-4. [CrossRef]

48. Guiffaut, C.; Rouvrais, N.; Reineix, A.; Pecqueux, B. Insulated Oblique Thin Wire Formalism in the FDTD Method. IEEE Trans. Electromagn. Compat. 2017, 59, 1532-1540. [CrossRef]

49. Liao, Z.P.; Wong, H.L.; Yang, B.P.; Yuan, Y.F. A transmitting boundary for transient wave analysis. Sci. Sin. Ser. A 1984, 27, 1063-1076.

50. Navarro, E.; Litva, J.; Wu, C.; Chung, P. Application of PML superabsorbing boundary condition to non-orthogonal FDTD method. Electron. Lett. 1994, 30, 1654-1656. [CrossRef]

51. Kraus, J. Helical Beam Antennas for Wide-Band Applications. Proc. IRE 1948, 36, 1236-1242. [CrossRef]

52. Kraus, J.D. Antennas, 2nd ed.; McGraw Hill: New York, NY, USA, 1988; pp. 265-338, ISBN 0-07-035422-7.

53. Sangary, N.T.; Nikolova, N.K. Line-of-sight approximation to the equivalence principle. IEEE Trans. Antennas Propag. 2004, 52, 1890-1897. [CrossRef] 